\title{
On the Spectrum of Differential Operators Under Riemannian Coverings
}

\author{
Panagiotis Polymerakis ${ }^{1}$
}

Received: 8 May 2018 / Published online: 30 April 2019

(c) The Author(s) 2019

\begin{abstract}
For a Riemannian covering $p: M_{2} \rightarrow M_{1}$, we compare the spectrum of an essentially self-adjoint differential operator $D_{1}$ on a bundle $E_{1} \rightarrow M_{1}$ with the spectrum of its lift $D_{2}$ on $p^{*} E_{1} \rightarrow M_{2}$. We prove that if the covering is infinite sheeted and amenable, then the spectrum of $D_{1}$ is contained in the essential spectrum of any self-adjoint extension of $D_{2}$. We show that if the deck transformations group of the covering is infinite and $D_{2}$ is essentially self-adjoint (or symmetric and bounded from below), then $D_{2}$ (or the Friedrichs extension of $D_{2}$ ) does not have eigenvalues of finite multiplicity and in particular, its spectrum is essential. Moreover, we prove that if $M_{1}$ is closed, then $p$ is amenable if and only if it preserves the bottom of the spectrum of some/any Schrödinger operator, extending a result due to Brooks.
\end{abstract}

Keywords Spectrum of differential operator - Amenable covering $\cdot$ Bottom of spectrum $\cdot$ Schrödinger operator

Mathematics Subject Classification 58J50 $\cdot$ 35P15 $\cdot$ 53C99

\section{Introduction}

A basic problem in Geometric Analysis is the investigation of relations between the geometry of a manifold and the spectrum of a differential operator on it. In this direction, it is natural to study the behavior of the spectrum under maps between Riemannian manifolds, which respect the geometry of the manifolds to some extent. In this paper, we deal with this problem for Riemannian coverings.

Let $p: M_{2} \rightarrow M_{1}$ be a Riemannian covering of connected manifolds with (possibly empty) smooth boundary. A Schrödinger operator $S_{1}$ on $M_{1}$ is an operator of the form $S_{1}=\Delta+V$, where $\Delta$ is the (non-negative definite) Laplacian and $V: M_{1} \rightarrow \mathbb{R}$ is

Panagiotis Polymerakis

polymerp@mpim-bonn.mpg.de

1 Max Planck Institute for Mathematics, Vivatsgasse 7, 53111 Bonn, Germany 
smooth and bounded from below. For such an operator $S_{1}$ on $M_{1}$, its lift on $M_{2}$ is the operator $S_{2}=\Delta+V \circ p$. The first results involving possibly infinite-sheeted coverings and establishing connections between properties of the covering and the (Dirichlet) spectra of $S_{1}$ and $S_{2}$ are related to the change of the bottom (that is, the minimum) of the spectrum and were proved by Brooks [6,7]. He showed that if the underlying manifold is complete, of finite topological type, without boundary and the covering is normal and amenable, then the bottom of the spectrum of the Laplacian is preserved. Bérard and Castillon [4] extended this result by showing that if the covering is amenable and the underlying manifold is complete with finitely generated fundamental group and without boundary, then the bottom of the spectrum of any Schrödinger operator is preserved. Recently, it was proved in [2] that the bottom of the spectrum of a Schrödinger operator is preserved under amenable coverings, without any topological or geometric assumptions.

In this paper, we prove a global result about this problem in a more general context. Instead of comparing the bottoms of the spectra, we prove inclusion of spectra under some reasonable assumptions. Moreover, our context allows us to impose various boundary conditions on Schrödinger operators (for instance, Dirichlet, Neumann, mixed, and Robin), while the former results involve only Dirichlet conditions. Furthermore, our theorems are applicable to a broad class of differential operators, including Schrödinger operators with magnetic potential (that is, first-order term), Dirac operators, higher-order Laplacian, and Laplace-type operators on vector bundles. It is worth to point out that the Hodge Laplacian is a special case of the latter ones, as well as the Jacobi (stability) operator of a minimal submanifold. Furthermore, in this context, we may consider the Laplacian on weighted manifolds (or Laplacian with density).

In order to simplify the statements of our results, we need to set up some notation. Consider a Riemannian or Hermitian vector bundle $E_{1} \rightarrow M_{1}$ endowed with a (not necessarily metric) connection $\nabla$. Let $D_{1}$ be a (not necessarily elliptic) differential operator of arbitrary order on $E_{1}$. We consider the pullback bundle $E_{2}:=p^{*} E_{1} \rightarrow M_{2}$ endowed with the corresponding metric and connection, and the lift $D_{2}$ of $D_{1}$.

If $M_{1}$ has empty boundary, we consider the space of compactly supported smooth sections of $E_{i}$ as the domain of $D_{i}, i=1,2$. If $M_{1}$ has non-empty boundary, as the domain of $D_{1}$ we consider the space of compactly supported smooth sections $\eta$ of $E_{1}$ satisfying a number of boundary conditions of the form

$$
\sum_{j=0}^{k} a_{j} \nabla_{n}^{(j)} \eta=0 \text { on } \partial M_{1},
$$

where $n$ is the inward pointing normal to $\partial M_{1}$ and $a_{j}$ 's are functions defined on $\partial M_{1}$. For example, in this context, we may impose boundary conditions of the form $\eta=\nabla_{n} \eta=\cdots=\nabla_{n}^{(k)} \eta=0$ on $\partial M_{1}$, for some $k \in \mathbb{N}$. As the domain of $D_{2}$ we consider the space of compactly supported, smooth sections of $E_{2}$ that satisfy analogous boundary conditions to the ones imposed on the domain of $D_{1}$.

Let $\mu_{1}$ be a measure expressed via a positive smooth density in terms of the volume element of $M_{1}$, that is, $\mathrm{d} \mu_{1}=h \mathrm{~d}$ Vol. Let $\mu_{2}$ be the corresponding measure on $M_{2}$, that is, $\mathrm{d} \mu_{2}=(h \circ p) \mathrm{d}$ Vol. We consider the operators $D_{i}$ restricted to the above domains as densely defined operators in $L^{2}\left(E_{i}, \mu_{i}\right), i=1,2$. 
For sake of simplicity, we present here special versions of our main results involving self-adjoint operators. The results are stated for infinite-sheeted coverings, since this is the interesting case of amenable coverings. However, we also prove the analogous results for finite-sheeted coverings. Our first result provides inclusion of the spectrum $\sigma\left(\bar{D}_{1}\right)$ of the closure of $D_{1}$, as long as it is self-adjoint, in the essential spectrum $\sigma_{\text {ess }}\left(D_{2}^{\prime}\right)$ of any self-adjoint extension $D_{2}^{\prime}$ of $D_{2}$.

Theorem 1.1 Assume that $D_{1}$ is essentially self-adjoint and let $D_{2}^{\prime}$ be a self-adjoint extension of $D_{2}$. If the covering is infinite sheeted and amenable, then the spectra of the operators satisfy $\sigma\left(\bar{D}_{1}\right) \subset \sigma_{\mathrm{ess}}\left(D_{2}^{\prime}\right)$.

Recall that a Schrödinger operator on a complete manifold is essentially self-adjoint on the space of compactly supported smooth functions vanishing on the boundary (if it is non-empty). Therefore, in the context of Schrödinger operators, it follows that if the underlying manifold is complete and the covering is infinite sheeted and amenable, then the spectrum of $S_{1}$ is contained in the essential spectrum of $S_{2}$.

An important case where the above theorem cannot be applied is that of Schrödinger operators on non-complete Riemannian manifolds. A Schrödinger operator on such a manifold does not have a unique self-adjoint extension, when restricted to the above domain, and we are interested in the spectrum of its Friedrichs extension. According to [2], if the covering is amenable, then the bottoms of the spectra of $S_{1}$ and $S_{2}$ coincide. The amenability is used only to establish $\lambda_{0}\left(S_{2}\right) \leq \lambda_{0}\left(S_{1}\right)$, since the inverse inequality holds for any covering, where $\lambda_{0}$ stands for the bottom of the spectrum. This motivates us to establish the following theorem, which compares the bottom $\lambda_{0}\left(D_{1}^{(F)}\right)$ of the spectrum of the Friedrichs extension of $D_{1}$ with the bottom $\lambda_{0}^{\text {ess }}\left(D_{2}^{(F)}\right)$ of the essential spectrum of the Friedrichs extension of $D_{2}$, when the operators are symmetric and bounded from below.

Theorem 1.2 Assume that $D_{i}$ is symmetric and bounded from below, and denote by $D_{i}^{(F)}$ its Friedrichs extension, $i=1$, 2. If the covering is infinite sheeted and amenable, then $\lambda_{0}^{\text {ess }}\left(D_{2}^{(F)}\right) \leq \lambda_{0}\left(D_{1}^{(F)}\right)$.

In particular, for Schrödinger operators, it follows that if the covering is infinite sheeted and amenable, then the bottom of the spectrum of $S_{1}$ is equal to the bottom of the essential spectrum of $S_{2}$, without any topological or geometric assumptions.

The above results involve amenable coverings. However, the deck transformations group of a (possibly non-amenable) covering provides information about the group of isometries of the covering space. This motivates us to work in a more general context than Riemannian coverings and prove that under some symmetry assumptions, an essentially self-adjoint differential operator does not have eigenvalues of finite multiplicity and in particular, its spectrum is essential. Moreover, we show the analogous result for the Friedrichs extension of a symmetric and bounded from below differential operator. In the context of Riemannian coverings, we obtain the following immediate consequences.

Corollary 1.3 Assume that $D_{2}$ is essentially self-adjoint. If the deck transformations group of the covering is infinite, then $\bar{D}_{2}$ does not have eigenvalues of finite multiplicity and in particular, $\sigma\left(\bar{D}_{2}\right)=\sigma_{\mathrm{ess}}\left(\bar{D}_{2}\right)$. 
Corollary 1.4 Assume that $D_{2}$ is symmetric and bounded from below, and denote by $D_{2}^{(F)}$ its Friedrichs extension. If the deck transformations group of the covering is infinite, then $D_{2}^{(F)}$ does not have eigenvalues of finite multyplicity and in particular, $\sigma\left(D_{2}^{(F)}\right)=\sigma_{\text {ess }}\left(D_{2}^{(F)}\right)$.

For Schrödinger operators, it follows that if the deck transformations group of the covering is infinite, then the spectrum of $S_{2}$ is essential, without any assumptions on the manifolds.

All the above results provide information about the spectra from properties of the covering (amenability or infinite deck transformations group). In the converse direction, Brooks [6] proved that if a normal Riemannian covering of a closed manifold (that is, compact without boundary) preserves the bottom of the spectrum of the Laplacian, then the covering is amenable. In this paper, we extend this result to Schrödinger operators and to not necessarily normal coverings. Recall that local isometries between complete Riemannian manifolds are (not necessarily normal) Riemannian coverings. In the following theorem, we denote by $h^{\text {ess }}(M)$ the supremum of the Cheeger's constants over complements of compact and smoothly bounded domains of $M$.

Theorem 1.5 Let $p: M_{2} \rightarrow M_{1}$ be a Riemannian covering with $M_{1}$ closed. Then the following are equivalent:

(i) $p$ is infinite sheeted and amenable,

(ii) $\sigma\left(S_{1}\right) \subset \sigma_{\text {ess }}\left(S_{2}\right)$ for some/any Schrödinger operator $S_{1}$ on $M_{1}$ and its lift $S_{2}$,

(iii) $\lambda_{0}\left(S_{1}\right)=\lambda_{0}^{\text {ess }}\left(S_{2}\right)$ for somelany Schrödinger operator $S_{1}$ on $M_{1}$ and its lift $S_{2}$,

(iv) $h^{\mathrm{ess}}\left(M_{2}\right)=0$.

It is worth to point out that Brooks proved his theorem in a quite complicated way, relying heavily on geometric measure theory. Our proof of the above theorem is significantly simpler and avoids the use of geometric measure theory. Moreover, this result yields that the assumption of amenability is natural in Theorems 1.1 and 1.2. Indeed, if we restrict ourselves to Schrödinger operators and coverings of closed manifolds, amenability is actually equivalent to the conclusions of these theorems.

Furthermore, Brooks [7], and more recently, Roblin and Tapie [22] proved that under some more general (but still quite restrictive) assumptions, if the bottom of the spectrum of the Laplacian is preserved, then the covering is amenable. In particular, these assumptions imply that the bottom of the spectrum of the Laplacian on $M_{1}$ is not in the essential spectrum. Moreover, Brooks [7] provided examples demonstrating that without these conditions, the bottom of the spectrum of the Laplacian may be preserved even if the covering is non-amenable. This suggests that under some assumptions on the geometry and the spectrum of the Laplacian on $M_{1}$, the bottom of the spectrum is preserved under a weaker assumption than amenability of the covering. In this direction, as an application of Theorem 1.1, we prove the following result.

Corollary 1.6 Let $p: M_{2} \rightarrow M_{1}$ be a Riemannian covering with $M_{1}$ complete. Let $S_{1}$ be a Schrödinger operator on $M_{1}$ with $\lambda_{0}\left(S_{1}\right) \in \sigma_{\mathrm{ess}}\left(S_{1}\right)$, and $S_{2}$ its lift on $M_{2}$. If there exists a compact $K \subset M_{1}$, such that the image of the fundamental group of any connected component of $M_{1} \backslash K$ in $\pi_{1}\left(M_{1}\right)$ is amenable, then $\lambda_{0}\left(S_{1}\right)=\lambda_{0}\left(S_{2}\right)$. 
The paper is organized as follows: In Sect. 2, we give some preliminaries. In Sects. 3 and 4, we present the construction which is used in order to prove Theorem 1.2 and a more general result (Theorem 4.1) than Theorem 1.1. The proofs are given in Sect. 4, where we also present the analogous results for finite-sheeted coverings. In Sect. 5, we study manifolds with high symmetry and establish Corollaries 1.3 and 1.4. In Sect. 6, we present an alternative proof of Brooks' Theorem [6], extending it to not necessarily normal Riemannian coverings. In Sect. 7, we introduce the notion of renormalized Schrödinger operators, which is used to prove Theorem 1.5. Moreover, in this section we establish Corollary 1.6 and we present a simple example demonstrating that the behavior of the bottom of the spectrum of the connection Laplacian under a covering depends on the corresponding metric connection. Therefore, a main point in our results is the independence from the vector bundles, the connections, and the differential operators.

\section{Preliminaries}

We first recall some basic facts from functional analysis. For more details, see [17]. Let $A: \mathcal{D}(A) \subset \mathcal{H} \rightarrow \mathcal{H}$ be a closed (linear) operator on a separable Hilbert space $\mathcal{H}$ over a field $\mathbb{F}$, where $\mathbb{F}=\mathbb{R}$ or $\mathbb{F}=\mathbb{C}$. The spectrum of $A$ is given by

$$
\sigma(A):=\{\lambda \in \mathbb{F}:(A-\lambda): \mathcal{D}(A) \rightarrow \mathcal{H} \text { not bijective }\}
$$

The essential spectrum of $A$ is defined as

$$
\sigma_{\mathrm{ess}}(A):=\{\lambda \in \mathbb{F}:(A-\lambda): \mathcal{D}(A) \rightarrow \mathcal{H} \text { not Fredholm }\}
$$

Recall that an operator is called Fredholm if its kernel is finite-dimensional and its range is closed and of finite codimension. The discrete spectrum of $A$ is the complement of the essential spectrum in the spectrum of $A$, that is, $\sigma_{d}(A):=\sigma(A) \backslash \sigma_{\mathrm{ess}}(A)$.

The approximate point spectrum of $A$, denoted by $\sigma_{\text {ap }}(A)$, is defined as the set of all $\lambda \in \mathbb{F}$, such that there exists $\left(v_{k}\right)_{k \in \mathbb{N}} \subset \mathcal{D}(A)$ with $\left\|v_{k}\right\|=1$ and $(A-\lambda) v_{k} \rightarrow 0$ in $\mathcal{H}$. For $\lambda \in \mathbb{F}$, a Weyl sequence for $A$ and $\lambda$ is a sequence $\left(v_{k}\right)_{k \in \mathbb{N}} \subset \mathcal{D}(A)$, such that $\left\|v_{k}\right\|=1, v_{k} \rightarrow 0$ and $(A-\lambda) v_{k} \rightarrow 0$ in $\mathcal{H}$, where " $\rightarrow$ " denotes the weak convergence in $\mathcal{H}$. The Weyl spectrum of $A$, denoted by $\sigma_{W}(A)$, is the set of all $\lambda \in \mathbb{F}$, such that there exists a Weyl sequence for $A$ and $\lambda$.

The following proposition is the characterization of the spectrum of a self-adjoint operator as the set of approximate eigenvalues and the well-known Weyl's criterion for the essential spectrum.

Proposition 2.1 If $A$ is self-adjoint, then $\sigma_{\mathrm{ap}}(A)=\sigma(A), \sigma_{W}(A)=\sigma_{\mathrm{ess}}(A)$ and $\sigma_{d}(A)$ consists of isolated eigenvalues of A of finite multiplicity. In particular, $\sigma_{\mathrm{ess}}(A)$ consists of eigenvalues of $A$ of infinite multiplicity and accumulation points of $\sigma(A)$.

Since we are interested in closures of operators, we need the following elementary lemma, characterizing the approximate point spectrum and the Weyl spectrum of the closure in terms of the initial operator. 
Lemma 2.2 Assume that $A$ is the closure of an operator $B: \mathcal{D}(B) \subset \mathcal{H} \rightarrow \mathcal{H}$ and consider $\lambda \in \mathbb{F}$. Then:

(i) $\lambda \in \sigma_{\mathrm{ap}}(A)$ if and only if there exists $\left(v_{k}\right)_{k \in \mathbb{N}} \subset \mathcal{D}(B)$, such that $\left\|v_{k}\right\|=1$ and $(B-\lambda) v_{k} \rightarrow 0$ in $\mathcal{H}$

(ii) $\lambda \in \sigma_{W}(A)$ if and only if there exists $\left(v_{k}\right)_{k \in \mathbb{N}} \subset \mathcal{D}(B)$, such that $\left\|v_{k}\right\|=1, v_{k} \rightarrow 0$ and $(B-\lambda) v_{k} \rightarrow 0$ in $\mathcal{H}$.

For an operator $B: \mathcal{D}(B) \subset \mathcal{H} \rightarrow \mathcal{H}$ and $v \in \mathcal{D}(B) \backslash\{0\}$, the Rayleigh quotient of $v$ with respect to $B$ is defined as

$$
\mathcal{R}_{B}(v):=\frac{\langle B v, v\rangle}{\|v\|^{2}} .
$$

If $B$ is symmetric, then $\mathcal{R}_{B}(v) \in \mathbb{R}$, for any $v \in \mathcal{D}(B) \backslash\{0\}$, and $B$ is bounded from below if the infimum of $\mathcal{R}_{B}(v)$, with $v \in \mathcal{D}(B) \backslash\{0\}$, is finite. In this case, this infimum is called the lower bound of $B$.

The spectrum of a self-adjoint operator $A$ is contained in $\mathbb{R}$ and the bottom (that is, the minimum) of the spectrum and the bottom of the essential spectrum of $A$ are denoted by $\lambda_{0}(A)$ and $\lambda_{0}^{\text {ess }}(A)$, respectively. The following characterization of the bottom of the spectrum is due to Rayleigh.

Proposition 2.3 If $A: \mathcal{D}(A) \subset \mathcal{H} \rightarrow \mathcal{H}$ is self-adjoint, then

$$
\lambda_{0}(A)=\inf _{v \in \mathcal{D}(A) \backslash\{0\}} \mathcal{R}_{A}(v) .
$$

If, in addition, $A$ is the closure of an operator $B: \mathcal{D}(B) \subset \mathcal{H} \rightarrow \mathcal{H}$, then the bottom of the spectrum of $A$ is given by

$$
\lambda_{0}(A)=\inf _{v \in \mathcal{D}(B) \backslash\{0\}} \mathcal{R}_{B}(v)
$$

Throughout the paper, manifolds are connected, with possibly empty, smooth, and not necessarily connected boundary, unless otherwise stated. Let $p: M_{2} \rightarrow M_{1}$ be a Riemannian covering of $m$-dimensional manifolds, $E_{1} \rightarrow M_{1}$ a Riemannian or Hermitian vector bundle of rank $\ell$, and $D_{1}: \Gamma\left(E_{1}\right) \rightarrow \Gamma\left(E_{1}\right)$ a differential operator of order $d$. Consider the pullback bundle $E_{2}:=p^{*} E_{1}$ on $M_{2}, y \in M_{2}$ and set $x:=p(y)$. Let $U_{2}$ be an open neighborhood of $y$, such that the restriction $\left.p\right|_{U_{2}}$ is an isometry onto its image $U_{1}$. The lift $D_{2}: \Gamma\left(E_{2}\right) \rightarrow \Gamma\left(E_{2}\right)$ of $D_{1}$ is the differential operator defined by

$$
D_{2} \eta(z):=\left(\left.p\right|_{U_{2}}\right)^{*}\left(D_{1}\left(\left(\left.p\right|_{U_{2}} ^{-1}\right)^{*} \eta\right)(p(z))\right)
$$

for any $\eta \in \Gamma\left(E_{2}\right)$ and $z \in U_{2}$. Without loss of generality, we may assume that $U_{1}$ is contained in a coordinate neighborhood and there exists a local trivialization $\left.E_{1}\right|_{U_{1}} \rightarrow U_{1} \times \mathbb{F}^{\ell}$. With respect to this coordinate system and trivialization, $D_{1}$ is expressed as 


$$
D_{1}=\sum_{|\alpha| \leq d} A^{\alpha} \frac{\partial^{|\alpha|}}{\partial x^{\alpha}}
$$

where $A^{\alpha}$ are smooth maps defined on $U_{1}$, with values $\ell \times \ell$ matrices with entries in $\mathbb{F}$. Then, with respect to the lifted coordinate system and the corresponding trivialization $\left.E_{2}\right|_{U_{2}} \rightarrow U_{2} \times \mathbb{F}^{\ell}, D_{2}$ has the local expression

$$
D_{2}=\sum_{|\alpha| \leq d}\left(A^{\alpha} \circ p\right) \frac{\partial^{|\alpha|}}{\partial y^{\alpha}}
$$

Let $M$ be a Riemannian manifold and $E \rightarrow M$ a Riemannian or Hermitian vector bundle endowed with a connection $\nabla$. Assume that $M$ has non-empty boundary, denote by $n$ the inward pointing normal to $\partial M$, and extend $n$ locally as the velocity of unit speed geodesics normal to the boundary. For $\eta \in \Gamma_{c}(E)$ and $k \in \mathbb{N}$, consider the following sections defined in a neighborhood of the boundary

$$
\nabla_{n}^{(k)} \eta:=\nabla_{n}\left(\nabla_{n}^{(k-1)} \eta\right), \text { where } \nabla_{n}^{(0)} \eta:=\eta .
$$

Similarly, for $f \in C_{c}^{\infty}(M)$ and $k \in \mathbb{N}$, consider the following functions defined in a neighborhood of the boundary

$$
n^{(k)}(f):=n\left(n^{(k-1)} f\right), \text { where } n^{(0)} f:=f .
$$

Lemma 2.4 Let $M$ be a Riemannian manifold, $E \rightarrow M$ a Riemannian or Hermitian vector bundle endowed with a connection $\nabla$ and $D: \Gamma(E) \rightarrow \Gamma(E)$ a differential operator. If $M$ has emptyboundary, $\operatorname{set} \mathcal{D}(D):=\Gamma_{c}(E)$. If $M$ has non-empty boundary, consider $v \in \mathbb{N}$, and for $l=1, \ldots, v$, let $k_{l} \in \mathbb{N}$ and $a_{j, l}$ be real or complex-valued functions (depending on whether $E$ is Riemannian or Hermitian) defined on $\partial M$, $j=0, \ldots, k_{l}$. Let $n$ be the inward pointing normal to $\partial M$ and consider

$$
\mathcal{D}(D):=\left\{\eta \in \Gamma_{c}(E): \sum_{j=0}^{k_{l}} a_{j, l} \nabla_{n}^{(j)} \eta=0 \text { on } \partial M, l=1, \ldots, \nu\right\} .
$$

Let $\mu$ be a measure on $M$ expressed via a positive smooth density with respect to the volume element of $M$; that is, there exists a positive $h \in C^{\infty}(M)$, such that $d \mu=h d$ Vol. Then the operator $D: \mathcal{D}(D) \subset L^{2}(E, \mu) \rightarrow L^{2}(E, \mu)$ is closable.

Proof Consider the formal adjoint $D^{\text {ad }}$ of $D$ in $L^{2}(E)$, defined by

$$
\langle D \eta, \theta\rangle_{L^{2}(E)}=\left\langle\eta, D^{\mathrm{ad}} \theta\right\rangle_{L^{2}(E)},
$$

for all $\eta \in \mathcal{D}(D)$ and $\theta \in \Gamma_{c c}(E)$, where $\Gamma_{c c}(E)$ is the space of smooth sections, compactly supported in the interior of $M$. Evidently, for $\eta \in \mathcal{D}(D)$ and $\theta \in \Gamma_{c c}(E)$, we have 


$$
\langle D \eta, \theta\rangle_{L^{2}(E, \mu)}=\left\langle\eta, D^{\prime} \theta\right\rangle_{L^{2}(E, \mu)} \text {, where } D^{\prime} \theta:=\frac{1}{h} D^{\mathrm{ad}}(h \theta) .
$$

It is clear that the operator $D^{\prime}: \Gamma_{c c}(E) \subset L^{2}(E, \mu) \rightarrow L^{2}(E, \mu)$ is densely defined and its adjoint satisfies $D \subset\left(D^{\prime}\right)^{*}$. Since the adjoint is closed, it follows that $D$ is closable.

A Schrödinger operator on a possibly non-connected Riemannian manifold $M$ is an operator of the form $S:=\Delta+V$, where $\Delta$ is the Laplacian and $V: M \rightarrow \mathbb{R}$ is smooth and bounded from below. If $M$ is complete and without boundary, then $S$ is essentially self-adjoint on $C_{c}^{\infty}(M)$, that is, the closure of $S: C_{c}^{\infty}(M) \subset L^{2}(M) \rightarrow L^{2}(M)$ is self-adjoint. If $M$ is complete with non-empty boundary, then $S$ is essentially selfadjoint on $\left\{f \in C_{c}^{\infty}(M): f=0\right.$ on $\left.\partial M\right\}$. If $M$ is non-complete, then $S$ restricted to the above domain does not have a unique self-adjoint extension, and we are interested in the Friedrichs extension of $S$. By abuse of notation, the spectrum and the essential spectrum of the above- described self-adjoint operator are denoted by $\sigma(S)$ and $\sigma_{\text {ess }}(S)$, respectively, and their bottoms by $\lambda_{0}(S)$ and $\lambda_{0}^{\text {ess }}(S)$, respectively. These sets and quantities for the Laplacian on $M$ are denoted by $\sigma(M), \sigma_{\text {ess }}(M)$ and $\lambda_{0}(M), \lambda_{0}^{\text {ess }}(M)$, respectively.

Let $p: M_{2} \rightarrow M_{1}$ be a Riemannian covering of complete manifolds without boundary. For $x \in M_{1}$ and $y \in p^{-1}(x)$, the fundamental domain of $p$ centered at $y$ is defined by

$$
D_{y}:=\left\{z \in M_{2}: d(z, y) \leq d\left(z, y^{\prime}\right) \text { for all } y^{\prime} \in p^{-1}(x)\right\}
$$

Some basic properties of these fundamental domains are presented in [2]. It is clear that $D_{y}$ is closed and $M_{2}$ is the union of $D_{y}$, with $y \in p^{-1}(x)$. It is worth to point out that the intersection of different fundamental domains is of measure zero. Moreover, $\partial D_{y}$ and the cut locus $\operatorname{Cut}(x)$ of $x$ are of measure zero and $p: D_{y} \backslash \partial D_{y} \rightarrow M_{1} \backslash C_{0}$ is an isometry, where $C_{0}$ is a subset of $\operatorname{Cut}(x)$. The following two lemmas are proved in [2]. The lemma after these is proved similarly to Lemma 2.6. In these lemmas and in the sequel, we denote open and closed balls by $B$ and $C$, respectively.

Lemma 2.5 If $K \subset B(x, r)$, then $p^{-1}(K) \cap D_{y} \subset B(y, r)$. In particular, if $K$ is compact, then $p^{-1}(K) \cap D_{y}$ is compact.

Lemma 2.6 For any $r>0$, there exists $N(r) \in \mathbb{N}$, such that any $z \in M_{2}$ is contained in at most $N(r)$ of the balls $C(y, r)$, with $y \in p^{-1}(x)$.

Lemma 2.7 Consider the universal coverings $p_{i}: \tilde{M} \rightarrow M_{i}, i=1,2$. For any $r$, $r_{0}>0$, there exists $\tilde{N}\left(r, r_{0}\right) \in \mathbb{N}$, such that

$$
\#\left\{w \in p_{2}^{-1}(z): B\left(w, r_{0}\right) \cap C(u, r) \neq \emptyset\right\} \leq \tilde{N}\left(r, r_{0}\right)
$$

for all $u \in p_{1}^{-1}(x)$ and $z \in M_{2}$.

It is worth to point out that the quantities $N(r)$ and $\tilde{N}\left(r, r_{0}\right)$ in the above lemmas depend on the choice of $x \in M_{1}$. 
Finally, we recall the notions of amenable right action and amenable covering. For more details on amenable left actions, which are completely analogous to right actions, see [4, Sect. 2]. A right action of a countable group $\Gamma$ on a countable set $X$ is called amenable if there exists a $\Gamma$-invariant mean on $L^{\infty}(X)$. The following characterization is due to Følner.

Proposition 2.8 The right action of a countable group $\Gamma$ on a non-empty, countable set $X$ is amenable if and only if for any finite $G \subset \Gamma$ and $\varepsilon>0$, there exists a non-empty, finite $F \subset X$, such that

$$
\#(F \backslash F g)<\varepsilon \#(F),
$$

for all $g \in G$. Such a set $F$ is called a Følner set for $G$ and $\varepsilon$.

A countable group $\Gamma$ is called amenable if the right action of $\Gamma$ on itself is amenable. In this case, the right action of $\Gamma$ on any countable set $X$ is amenable. Moreover, it is clear that any right action on a non-empty, finite set is amenable.

A Riemannian covering $p: M_{2} \rightarrow M_{1}$ is called amenable if the right action of $\pi_{1}\left(M_{1}\right)$ on $\pi_{1}\left(M_{2}\right) \backslash \pi_{1}\left(M_{1}\right)$ (that is, the set of right cosets of $\pi_{1}\left(M_{2}\right)$ in $\pi_{1}\left(M_{1}\right)$, when considered as deck transformations groups of the universal coverings) is amenable. Clearly, a normal covering is amenable if and only if its deck transformations group is amenable. Furthermore, finite-sheeted coverings are amenable.

The following criteria for amenability of groups are immediate consequences of the definition and Proposition 2.8.

Corollary 2.9 Any finitely generated group of subexponential growth is amenable.

Corollary 2.10 A countable group $\Gamma$ is amenable if and only if any finitely generated subgroup of $\Gamma$ is amenable

Corollary 2.11 Any countable solvable group is amenable.

Proof From Corollaries 2.9 and 2.10, it follows that any countable abelian group is amenable. From the definition, it is clear that an extension of an amenable group by an amenable group is also amenable.

\section{Coverings of Manifolds with Boundary}

The aim of this section is to show the following proposition, according to which, any Riemannian covering of manifolds with boundary can be "extended" to a Riemannian covering of manifolds without boundary.

Proposition 3.1 Let $M$ be a Riemannian manifold with non-empty boundary. Then there exists a Riemannian manifold $N$ of the same dimension, without boundary and an isometric embedding $i: M \rightarrow N$, such that, after identifying $M$ with $i(M)$, any Riemannian covering $p: M^{\prime} \rightarrow M$ can be extended to a Riemannian covering $p: N^{\prime} \rightarrow N$. 
In order to prove this proposition, we need to establish some auxiliary lemmas.

Lemma 3.2 Let $M$ be a Riemannian manifold with non-empty boundary. Then there exists a Riemannian manifold $N$ of the same dimension, without boundary, an isometric embedding $i: M \rightarrow N$, and a strong deformation retraction of $N$ onto $i(M)$.

Proof Consider the space $\partial M \times[0,+\infty)$ and the map $\Psi: \partial M \rightarrow \partial M \times[0,+\infty)$, defined by $\Psi(x):=(x, 0)$. Then $N:=M \cup_{\Psi}(\partial M \times[0,+\infty))$ is a smooth manifold and there exists a smooth embedding $i: M \rightarrow N$. Therefore, $M$ can be identified with $i(M)$. Since $M$ is connected, so is $N$, and there exists a strong deformation retraction of $N$ onto $M$, obtained by considering $F_{t}(x, r):=(x,(1-t) r)$ in the glued ends $\partial \times[0,+\infty)$.

It remains to extend the Riemannian metric of $M$ to a Riemannian metric of $N$. Any $x \in \partial M$ has an open neighborhood $U_{x}$ in $N$, such that there exists a smooth frame field $\left\{e_{1}, \ldots, e_{m}\right\}$ in $U_{x}$, where $m$ is the dimension of the manifolds. Let $g_{j k}:=\left\langle e_{j}, e_{k}\right\rangle$, $1 \leq j, k \leq m$, be the components of the Riemannian metric of $M$. Since they are smooth up to the boundary of $M$, they can be extended smoothly to a neighborhood of $x$. After passing to a smaller neighborhood of $x$ if needed, we may assume that $g_{j k}$ 's are smooth in $U_{x}$ and their matrix is symmetric and positive definite at any point of $U_{x}$. Hence, they express a Riemannian metric in $U_{x}$.

Clearly, $\partial M$ can be covered with such neighborhoods $U_{x}$. Consider the interior of $M$ as an open subset of $N$ endowed with its Riemannian metric and $N \backslash M$ with an arbitrary Riemannian metric. Combining these Riemannian metrics via a partition of unity subordinate to this open cover of $N$, gives rise to a Riemannian metric of $N$, which is an extension of the Riemannian metric of $M$.

Lemma 3.3 Let $M$ be a Riemannian manifold with non-empty boundary. Consider $N$ as in the previous lemma and identify $M$ with $i(M)$. Let $q: \tilde{N} \rightarrow N$ be the universal covering of $N$. Then the restriction $q: q^{-1}(M) \rightarrow M$ is the universal covering of $M$.

Proof Since there exists a strong deformation retraction of $N$ onto $M$, every loop in $N$ can be homotoped to a loop in $M$. This implies that for any $x \in M$ and $y_{1}, y_{2} \in q^{-1}(x)$, there exists a path in $q^{-1}(M)$ from $y_{1}$ to $y_{2}$. Since $M$ is connected, it follows that so is $q^{-1}(M)$ and the restriction $q: q^{-1}(M) \rightarrow M$ is a covering of (connected) manifolds.

Let $r_{M}: N \rightarrow M$ be a retraction. Then the map $r_{M} \circ q: \tilde{N} \rightarrow M$ is continuous and $r_{M} \circ q=q$ in $q^{-1}(M)$. From the Lifting Theorem, it follows that $r_{M} \circ q$ has a continuous lift $\tilde{r}_{M}: \tilde{N} \rightarrow q^{-1}(M)$, with $\tilde{r}_{M}\left(y_{0}\right)=y_{0}$, for some $y_{0} \in q^{-1}(M)$. Since $\left.\tilde{r}_{M}\right|_{q^{-1}(M)}$ is a deck transformation of the covering $q: q^{-1}(M) \rightarrow M$, it follows that $\tilde{r}_{M}: \tilde{N} \rightarrow q^{-1}(M)$ is a retraction. Since $\tilde{N}$ is simply connected, this yields that so is $q^{-1}(M)$.

Proof of Proposition 3.1: Consider $N$ and $q: \tilde{N} \rightarrow N$ as in the above lemmas, identify $M$ with $i(M)$ and set $\tilde{M}:=q^{-1}(M)$. Denote by $\Gamma_{N}$ and $\Gamma_{M}$ the deck transformations groups of $q: \tilde{N} \rightarrow N$ and $q: \tilde{M} \rightarrow M$, respectively. It is clear that for $g \in \Gamma_{N}$, we have $\left.g\right|_{\tilde{M}} \in \Gamma_{M}$, and any $\gamma \in \Gamma_{M}$ has a unique extension $\gamma^{\prime} \in \Gamma_{N}$. For any Riemannian covering $p: M^{\prime} \rightarrow M$, there exists a subgroup $\Gamma \subset \Gamma_{M}$, such that $M^{\prime}=\tilde{M} / \Gamma$. For $\Gamma^{\prime}:=\left\{\gamma^{\prime} \in \Gamma_{N}: \gamma \in \Gamma\right\}$ and $N^{\prime}:=\tilde{N} / \Gamma^{\prime}$, the inclusion $\tilde{M} \hookrightarrow \tilde{N}$ descends to an isometric embedding $M^{\prime} \rightarrow N^{\prime}$, which completes the proof. 


\section{Spectrum of Operators Under Amenable Coverings}

Throughout this section, we work in the following context, which is briefly described in the Introduction.

Let $p: M_{2} \rightarrow M_{1}$ be a Riemannian covering, $E_{1} \rightarrow M_{1}$ a Riemannian or Hermitian vector bundle endowed with a connection $\nabla$, and $D_{1}: \Gamma\left(E_{1}\right) \rightarrow \Gamma\left(E_{1}\right)$ a differential operator on $E_{1}$. Let $E_{2} \rightarrow M_{2}$ be the pullback bundle, endowed with the corresponding metric and connection $\nabla$, and $D_{2}: \Gamma\left(E_{2}\right) \rightarrow \Gamma\left(E_{2}\right)$ the lift of $D_{1}$. If $M_{1}$ has empty boundary, we consider the space of compactly supported smooth sections of $E_{i}$ as the domain of $D_{i}$, that is, $\mathcal{D}\left(D_{i}\right):=\Gamma_{c}\left(E_{i}\right), i=1,2$.

If $M_{1}$ has non-empty boundary, consider $v \in \mathbb{N}$, and for $l=1, \ldots, v$, let $k_{l} \in \mathbb{N}$ and $a_{j, l}^{(1)}$ be real or complex-valued functions (depending on whether $E_{1}$ is Riemannian or Hermitian) defined on $\partial M_{1}, j=0, \ldots, k_{l}$. It is worth to point out that we do not impose any assumptions on the functions $a_{j, l}^{(1)}$. Let $n_{i}$ be the inward pointing normal to $\partial M_{i}$, set $a_{j, l}^{(2)}:=a_{j, l}^{(1)} \circ p$, and consider

$$
\mathcal{D}\left(D_{i}\right):=\left\{\eta \in \Gamma_{c}\left(E_{i}\right): \sum_{j=0}^{k_{l}} a_{j, l}^{(i)} \nabla_{n_{i}}^{(j)} \eta=0 \text { on } \partial M_{i}, l=1, \ldots, v\right\}, i=1,2 .
$$

Let $\mu_{1}$ be a measure on $M_{1}$ expressed via a positive smooth density with respect to the volume element of $M_{1}$; that is, there exists a positive $h \in C^{\infty}\left(M_{1}\right)$, such that $d \mu_{1}=h d$ Vol. Let $\mu_{2}$ be the corresponding measure on $M_{2}$, i.e., $d \mu_{2}=(h \circ p) d$ Vol. We consider the operator $D_{i}$ as a densely defined operator

$$
D_{i}: \mathcal{D}\left(D_{i}\right) \subset L^{2}\left(E_{i}, \mu_{i}\right) \rightarrow L^{2}\left(E_{i}, \mu_{i}\right),
$$

$i=1,2$. When we refer to closability, symmetry, or essential self-adjointness of $D_{i}$, we consider the operator as in (2), $i=1,2$. From Lemma 2.4, the operator $D_{i}$ is closable and we denote by $\bar{D}_{i}$ its closure, $i=1,2$.

Our aim in this section is to prove Theorem 1.2 and the following more general version of Theorem 1.1.

Theorem 4.1 Let $D_{2}^{\prime}$ be a closed extension of $D_{2}$. If the covering is infinite sheeted and amenable, then $\sigma_{\text {ap }}\left(\bar{D}_{1}\right) \subset \sigma_{W}\left(D_{2}^{\prime}\right)$.

For convenience of the reader, we briefly describe the outline of the proof of this theorem. Given $\eta \in \mathcal{D}\left(D_{1}\right)$ with $\|\eta\|_{L^{2}\left(E_{1}, \mu_{1}\right)}=1, \lambda \in \mathbb{F}$, and $\varepsilon>0$, we want to prove that there exists $\zeta \in \mathcal{D}\left(D_{2}\right)$, such that

$$
\frac{\left\|\left(D_{2}-\lambda\right) \zeta\right\|_{L^{2}\left(E_{2}, \mu_{2}\right)}}{\|\zeta\|_{L^{2}\left(E_{2}, \mu_{2}\right)}} \leq\left\|\left(D_{1}-\lambda\right) \eta\right\|_{L^{2}\left(E_{1}, \mu_{1}\right)}+\varepsilon
$$

First consider the case where the manifolds are complete without boundary. Then there exists $r>0$, such that supp $\eta \subset B(x, r)$, for some $x \in M_{1}$. For $y \in p^{-1}(x)$, we consider a function $\varphi_{y} \in C_{c}^{\infty}\left(M_{2}\right)$ centered at $y$, whose profile is essentially 
independent from $y$. For a finite subset $P$ of $p^{-1}(x)$, we consider the test section $\chi \theta \in \mathcal{D}\left(D_{2}\right)$, where $\theta$ is the lift of $\eta$ and $\chi=\sum_{y \in P} \varphi_{y}$. For such a section we establish pointwise estimates of the form $\left\|\left(D_{2}-\lambda\right)(\chi \theta)\right\| \leq C$ in $M_{2}$, where $C$ is a constant independent from $P$.

Consider $p^{-1}(x)$ as a discrete graph, where two points are connected if their distance is less than $2 r+2$. For a point $y \in P \backslash \partial P$, it follows that $\chi \theta=\theta$ in $B(y, r)$. Moreover, $\chi \theta$ is supported in the union of the balls $B(y, r)$, with $y$ in $P$ or $y$ connected to some point in $\partial P$. From Lemma 2.6, there are at most $N(2 r+2) \#(\partial P)$ many $y \in p^{-1}(x)$ that are connected to some point in $\partial P$.

Since the covering is amenable, it follows that there exist finite subsets $P$ of $p^{-1}(x)$ with arbitrarily small isoperimetric ratio. Hence, the corresponding sections $\chi \theta$ coincide with $\theta$ in a relatively large part of their supports, while in the rest of their supports they satisfy the aforementioned estimates, which are independent from $P$. Therefore, the corresponding test sections $\chi \theta$ satisfy (3). Moreover, since $p$ is infinite sheeted, given a compact $K \subset M_{2}$, we may choose a finite $P \subset p^{-1}(x)$, so that $\chi \theta$ satisfies (3) and $\operatorname{supp}(\chi \theta)$ does not intersect $K$. This completes the proof of the theorem in case the manifolds are complete without boundary.

If the manifolds are non-complete without boundary, then we consider conformal Riemannian metrics that make the manifolds complete, and exploit the method described above.

If the manifolds have non-empty boundary, then we extend the given Riemannian covering to a Riemannian covering of manifolds without boundary, according to Proposition 3.1. Then we consider conformal Riemannian metrics that make the manifolds complete and exploit a slight variation of the above method. In this case, it is important to require that this new Riemannian metric on $M_{1}$ coincides with the original Riemannian metric in a compact neighborhood of supp $\eta$, so that this construction respects the imposed boundary conditions; that is, if $\eta \in \mathcal{D}\left(D_{1}\right)$, then $\chi \theta \in \mathcal{D}\left(D_{2}\right)$, for any finite subset $P$ of $p^{-1}(x)$.

\subsection{Partition of Unity}

In this subsection, we construct a special partition of unity, which is used in the sequel to obtain cut-off functions on $M_{2}$.

Let $K_{0}$ be a compact subset of $M_{1}$. Consider the universal coverings $p_{i}: \tilde{M} \rightarrow M_{i}$ and denote by $\Gamma_{i}$ the deck transformations group of $p_{i}, i=1,2$. If $M_{1}$ has empty boundary, consider a Riemannian metric $\mathfrak{h}_{1}$, conformal to the original metric $\mathfrak{g}_{1}$, such that $\left(M_{1}, \mathfrak{h}_{1}\right)$ is complete. Such a metric exists according to [21].

If $M_{1}$ has non-empty boundary, let $n_{i}$ be the inward pointing normal to $\partial M_{i}$, $i=1,2$, and $\tilde{n}$ the inward pointing normal to $\partial \tilde{M}$. Consider a Riemannian manifold $\left(N_{1}, \mathfrak{g}_{1}\right)$ containing $M_{1}$, as in Proposition 3.1, and a Riemannian metric $\mathfrak{h}_{1}$, conformal to the original metric $\mathfrak{g}_{1}$, such that $\left(N_{1}, \mathfrak{h}_{1}\right)$ is complete. Since $K_{0}$ is compact, we may assume that $\mathfrak{h}_{1}$ coincides with $\mathfrak{g}_{1}$ in a compact neighborhood of $K_{0}$. From Proposition 3.1, it follows that the Riemannian covering $p: M_{2} \rightarrow M_{1}$ can be extended to a Riemannian covering $p: N_{2} \rightarrow N_{1}$. Moreover, according to Lemma 3.3, $\tilde{M}$ can be identified with a domain of the simply connected covering space $\tilde{N}$ of $N_{1}$. 
From now on, geodesics are considered with respect to $\mathfrak{h}_{1}$ and its lifts. If $M_{1}$ has empty boundary, distances are considered with respect to $\mathfrak{h}_{1}$ or its lifts. In this case, we denote the open (respectively, closed) ball of radius $r$ around a point $z$ by $B(z, r)$ (respectively, $C(z, r)$ ). If $M_{1}$ has non-empty boundary, the distance between two points is considered in $\left(N_{1}, \mathfrak{h}_{1}\right)$ or its corresponding covering space. In this case, $B(z, r)$ and $C(z, r)$ stand for the corresponding balls in $M_{1}, M_{2}$, or $\tilde{M}$. For example, for $u \in \tilde{M}$ and $r>0$, we have

$$
B(u, r)=\{z \in \tilde{M}: d(z, u)<r\}
$$

where $d(\cdot, \cdot)$ is the distance function of $\tilde{N}$ induced by the lift of $\mathfrak{h}_{1}$.

Fix $x \in M_{1}, u \in p_{1}^{-1}(x)$, and $r>0$, such that $K_{0} \subset B(x, r)$. If $M_{1}$ has non-empty boundary, consider $r$ large enough, so that $B(u, r) \cap \partial \tilde{M} \neq \emptyset$.

Lemma 4.2 There exists a non-negative $\psi_{u} \in C_{c}^{\infty}(\tilde{M})$, with supp $\psi_{u} \subset C(u, r+1)$ and $\psi_{u}=1$ in $C(u, r+1 / 2)$. Moreover, if $M_{1}$ has non-empty boundary, $\psi_{u}$ can be chosen such that $\tilde{n}^{(i)} \psi_{u}=0$ on $\partial \tilde{M} \cap p_{1}^{-1}\left(K_{0}\right)$, for any $i \in \mathbb{N}$.

Proof It is clear that there exists a non-negative $\psi_{u}^{\prime} \in C_{c}^{\infty}(\tilde{M})$ with supp $\psi_{u}^{\prime} \subset$ $C(u, r+1)$ and $\psi_{u}^{\prime}=1$ in $C(u, r+1 / 2)$. If $M_{1}$ has empty boundary, this is the desired function. Otherwise, let $K:=\partial \tilde{M} \cap C(u, r+2)$ and denote by $n$ the inward pointing normal to $\partial \tilde{M}$ with respect to the lift of $\mathfrak{h}_{1}$. Since $K$ is compact, there exists $\varepsilon>0$, with $\varepsilon<1 / 8$, such that the map $\Phi: K \times[0,2 \varepsilon) \rightarrow \tilde{M}$, defined by $\Phi(z, t):=\exp _{z}(t n)$ is a diffeomorphism onto its image $K_{\varepsilon}$. Let $K_{1}:=\partial \tilde{M} \cap C(u, r+1 / 2+2 \varepsilon)$ and $K_{2}:=\partial \tilde{M} \cap C(u, r+1-2 \varepsilon)$. Clearly, there exists a non-negative $\tau \in C_{c}^{\infty}(\partial \tilde{M})$, with supp $\tau \subset K_{2}$ and $\tau=1$ in $K_{1}$. Extend it to $\tau^{\prime}$ in $K_{\varepsilon}$ by $\tau^{\prime}(\Phi(z, t)):=\tau(z)$, for all $(z, t) \in K \times[0,2 \varepsilon)$. Consider a smooth $f: \mathbb{R} \rightarrow \mathbb{R}$, with $0 \leq f \leq 1$, $f(t)=1$ for $t \leq \varepsilon$, and $f(t)=0$ for $t \geq 3 \varepsilon / 2$, and the function $v$ defined in $K_{\varepsilon}$ by $v(\Phi(z, t))=f(t)$, for all $(z, t) \in K \times[0,2 \varepsilon)$. Extend $v$ by zero outside $K_{\varepsilon}$ and set

$$
\psi_{u}:=v \tau^{\prime}+(1-v) \psi_{u}^{\prime} .
$$

Since $\operatorname{supp}\left(v \tau^{\prime}\right) \subset C(u, r+1), \operatorname{supp} \psi_{u}^{\prime} \subset C(u, r+1)$, it follows that supp $\psi_{u} \subset$ $C(u, r+1)$. Since $\varepsilon<1 / 8$, the points where $v$ is not smooth are not in $C(u, r+1)$, which yields that $\psi_{u} \in C_{c}^{\infty}(\tilde{M})$. Since $\psi_{u}^{\prime}=1$ in $C(u, r+1 / 2)$ and $\tau^{\prime}=1$ in $C(u, r+1 / 2) \cap K_{\varepsilon}$, it follows that $\psi_{u}=1$ in $C(u, r+1 / 2)$. In $\Phi(K \times[0, \varepsilon))$, which is a neighborhood of supp $\psi_{u} \cap \partial \tilde{M}$, we have $\psi_{u}=\tau^{\prime}$. In particular, in a neighborhood of the boundary, $\psi_{u}$ is constant along geodesics (with respect to the lift of $\mathfrak{h}_{1}$ ) that are normal to the boundary. This yields that $n^{(i)} \psi_{u}=0$ on $\partial \tilde{M}$, for any $i \in \mathbb{N}$. Since $\mathfrak{h}_{1}$ coincides with $\mathfrak{g}_{1}$ in a compact neighborhood of $K_{0}$, it follows that $\tilde{n}^{(i)} \psi_{u}=0$ on $\partial \tilde{M} \cap p_{1}^{-1}\left(K_{0}\right)$, for any $i \in \mathbb{N}$.

Let $\psi_{u}$ be a function as in the above lemma and for any $y \in p^{-1}(x)$, consider $u(y) \in p_{2}^{-1}(y)$ and $g(y) \in \Gamma_{1}$, such that $u(y)=g(y) u$. Consider the functions $\psi_{u(y)}:=\psi_{u} \circ g(y)^{-1}$ in $\tilde{M}$ and $\psi_{y}$ in $M_{2}$ defined by 


$$
\psi_{y}(z):=\sum_{w \in p_{2}^{-1}(z)} \psi_{u(y)}(w)
$$

It is clear that $\psi_{y} \in C_{c}^{\infty}\left(M_{2}\right)$, supp $\psi_{y} \subset C(y, r+1)$, and $\psi_{y} \geq 1$ in $C(y, r+1 / 2)$, for any $y \in p^{-1}(x)$. Moreover, if $M_{1}$ has non-empty boundary, then $n_{2}^{(i)} \psi_{y}=0$ on $\partial M_{2} \cap p^{-1}\left(K_{0}\right)$, for any $y \in p^{-1}(x)$ and $i \in \mathbb{N}$. From Lemma 2.6, there exists $N(r+2) \in \mathbb{N}$, such that for any $z \in M_{2}$, the ball $B(z, 1)$ intersects at most $N(r+2)$ of the supports of $\psi_{y}$, with $y \in p^{-1}(x)$. Therefore, $\sum_{y \in p^{-1}(x)} \psi_{y}$ is locally a finite sum and hence, well defined and smooth.

If $M_{1}$ is compact, we choose $r$ large enough, so that $\sum_{y \in p^{-1}(x)} \psi_{y} \geq 1$ in $M_{2}$. In this case, set $\psi_{1}:=0$ in $M_{2}$. If $M_{1}$ is non-compact, consider $f_{1} \in C_{c}^{\infty}\left(M_{1}\right)$ with $0 \leq f_{1} \leq 1, f_{1}=1$ in $C(x, r)$, supp $f_{1} \subset B(x, r+1 / 2)$, and let $\psi_{1}$ be the lift of $1-f_{1}$ on $M_{2}$. Then $\psi_{1} \in C^{\infty}\left(M_{2}\right), \psi_{1} \geq 0$ in $M_{2}$ and $\psi_{1}=0$ in $C(y, r)$, for all $y \in p^{-1}(x)$. Evidently, $\psi_{1}+\sum_{y \in p^{-1}(x)} \psi_{y} \geq 1$ in $M_{2}$.

Consider the smooth partition of unity consisting of the functions

$$
\varphi_{1}:=\frac{\psi_{1}}{\psi_{1}+\sum_{y^{\prime} \in p^{-1}(x)} \psi_{y^{\prime}}} \text { and } \varphi_{y}:=\frac{\psi_{y}}{\psi_{1}+\sum_{y^{\prime} \in p^{-1}(x)} \psi_{y^{\prime}}},
$$

with $y \in p^{-1}(x)$.

Remark 4.3 It is evident that $\operatorname{supp} \varphi_{1}=\operatorname{supp} \psi_{1}$, and for any $y \in p^{-1}(x)$, we have $\operatorname{supp} \varphi_{y}=\operatorname{supp} \psi_{y}, \sum_{y^{\prime} \in p^{-1}(x)} \varphi_{y^{\prime}}=1$ in $C(y, r)$, and $\varphi_{y}>0$ in $C(y, r+1 / 2)$. Since $K_{0} \subset B(x, r)$, it follows that $\psi_{1}=0$ in a neighborhood of $p^{-1}\left(K_{0}\right)$. If $M_{1}$ has non-empty boundary, then $n_{2}^{(i)} \psi_{y}=0$ on $\partial M_{2} \cap p^{-1}\left(K_{0}\right)$, for any $y \in p^{-1}(x)$ and $i \in \mathbb{N}$. This yields that $n_{2}^{(i)} \varphi_{y}=0$ on $\partial M_{2} \cap p^{-1}\left(K_{0}\right)$, for all $y \in p^{-1}(x)$ and $i \in \mathbb{N}$.

Let $\eta \in \mathcal{D}\left(D_{1}\right)$ and $\theta \in \Gamma\left(E_{2}\right)$ be the lift of $\eta$. Fix $x \in M_{1}, u \in p_{1}^{-1}(x)$, and $r>0$, such that $K_{0}:=\operatorname{supp} \eta \subset B(x, r)$. If $M_{1}$ has non-empty boundary, we choose $r$ large enough, so that $B(u, r) \cap \partial \tilde{M} \neq \varnothing$. Consider a partition of unity associated with $K_{0}$, $x, u$ and $r$ as in (5) and for a finite $P \subset p^{-1}(x)$, set $\chi:=\sum_{y \in P} \varphi_{y}$.

Remark 4.4 Since $P$ is finite, it follows that $\chi \in C_{c}^{\infty}\left(M_{2}\right)$ and $\chi \theta \in \Gamma_{c}\left(E_{2}\right)$. If $M_{1}$ has empty boundary, this yields that $\chi \theta \in \mathcal{D}\left(D_{2}\right)$. If $M_{1}$ has non-empty boundary, from Remark 4.3, we have that $n_{2}^{(i)} \varphi_{y}=0$ on $\partial M_{2} \cap \operatorname{supp} \theta$, for any $y \in p^{-1}(x)$ and $i \in \mathbb{N}$. In particular, if $\eta$ satisfies a boundary condition of the form

$$
\sum_{j=0}^{k} a_{j} \nabla_{n_{1}}^{(j)} \eta=0 \text { on } \partial M_{1}
$$

then for $\chi \theta$ we have

$$
\sum_{j=0}^{k}\left(a_{j} \circ p\right) \nabla_{n_{2}}^{(j)}(\chi \theta)=\sum_{y \in P} \sum_{j=0}^{k} \varphi_{y}\left(a_{j} \circ p\right) \nabla_{n_{2}}^{(j)} \theta=0 \text { on } \partial M_{2} .
$$


Hence, $\chi \theta$ satisfies analogous boundary conditions to $\eta$. Since $\eta \in \mathcal{D}\left(D_{1}\right)$, it follows that $\chi \theta \in \mathcal{D}\left(D_{2}\right)$.

Proposition 4.5 There exists a constant $C$, independent from $P$, such that for any $z \in M_{2}$, we have $\left\|D_{2}(\chi \theta)(z)\right\| \leq C$.

It is worth to point out that the constant in this proposition, as well as the estimates in the sequel, depend on various choices we made in this construction. For instance, they depend on the conformal Riemannian metric $\mathfrak{h}_{1}$, on $r$ and on the choice of $\psi_{u}$. The main point of this proposition is that there is no dependence on $P$.

Proof Consider $\delta>0$, such that for any $x^{\prime} \in C(x, r+1)$, the ball $B\left(x^{\prime}, 2 \delta\right)$ is evenly covered and contained in a coordinate neighborhood, and $\left.E_{1}\right|_{B\left(x^{\prime}, 2 \delta\right)}$ is trivial. Let $x_{1}, \ldots, x_{k} \in C(x, r+1)$, such that the balls $B\left(x_{i}, \delta\right)$, with $1 \leq i \leq k$, cover $C(x, r+1)$. In any ball $B\left(x_{i}, 2 \delta\right), D_{1}$ has a local expression of the form (1), with $A^{\alpha}$ smooth. This yields that in $B\left(x_{i}, \delta\right), D_{1}$ is expressed in the form (1), with $A^{\alpha}$ smooth and bounded. For any such ball, we fix a coordinate system (which can be extended to the corresponding ball of radius $2 \delta)$ and a trivialization. Since $C(x, r+1)$ is covered by finitely many such balls, it follows that there exists $C_{1}>0$, such that in any of these balls, we have $\left\|A^{\alpha}\right\| \leq C_{1}$, for all multi-indices $\alpha$ of absolute value less or equal to the order $d$ of $D_{1}$.

Since $\eta$ is smooth and compactly supported in $B(x, r)$, there exists $C_{2}>0$, such that in any of these balls, denoting by $\left(\eta^{(1)}, \ldots, \eta^{(\ell)}\right)$ the local expression of $\eta$, we have that

$$
\left\|\frac{\partial^{|\alpha|}}{\partial x^{\alpha}}\left(\eta^{(1)}, \ldots, \eta^{(\ell)}\right)\right\| \leq C_{2},
$$

for all multi-indices $\alpha$ of absolute value less or equal to $d$, that is, we have uniform estimates up to order $d$ for $\eta$ (with respect to this system of trivializations). We lift these balls and the corresponding coordinate systems and trivializations to $M_{2}$ and $\tilde{M}$. Similarly, if $\psi_{1} \neq 0$, we obtain uniform estimates up to order $d$ for $f_{1}$, which yield uniform estimates up to order $d$ for $\psi_{1}$ (with respect to the lifted system on $M_{2}$ ).

Since $\psi_{u}$ is smooth and compactly supported in $C(u, r+1)$, which intersects finitely many balls of the lifted system on $\tilde{M}$, there exist uniform estimates up to order $d$ for $\psi_{u}$. Since $\psi_{u(y)}$ is a composition of $\psi_{u}$ with an element of $\Gamma_{1}$, we obtain the same uniform estimates up to order $d$ for $\psi_{u(y)}$, for all $u(y)$. Recall the definition of $\psi_{y}$ in (4). Consider a ball $B\left(z^{\prime}, \delta\right)$ of the lifted system on $M_{2}$, which intersects supp $\psi_{y}$, and the corresponding coordinate system. It is clear that for any $w \in p_{2}^{-1}\left(z^{\prime}\right)$, the lifted system on $\tilde{M}$ contains the ball $B(w, \delta)$ and the corresponding coordinate system. From Lemma 2.7, there exists $\tilde{N}(r+1, \delta) \in \mathbb{N}$, independent from $y$ and $z^{\prime}$, such that at most $\tilde{N}(r+1, \delta)$ such balls intersect the support of $\psi_{u(y)}$. Since we have uniform estimates up to order $d$ for $\psi_{u(y)}$, which are independent from $y \in p^{-1}(x)$, we obtain the same uniform estimates up to order $d$ for $\psi_{y}$, for all $y \in p^{-1}(x)$. From Lemma 2.6, it follows that at most $N(r+1+\delta)$ of the supports of $\psi_{y}$, with $y \in p^{-1}(x)$, intersect the open ball $B(z, \delta)$, for any $z \in M_{2}$. This yields that there exist uniform estimates up to order $d$ for $\psi_{1}+\sum_{y \in p^{-1}(x)} \psi_{y}$. 
Recall the definition of $\varphi_{y}$ in (5). Since the denominator is greater or equal to 1 and we have uniform estimates (independent from $y$ ) up to order $d$ for the numerator and the denominator, we obtain the same uniform estimates up to order $d$ for $\varphi_{y}$, for all $y \in p^{-1}(x)$. From Lemma 2.6, at most $N(r+1+\delta)$ of the supports of $\varphi_{y}$, with $y \in p^{-1}(x)$, intersect the ball $B(z, \delta)$, for any $z \in M_{2}$. Therefore, we obtain uniform estimates up to order $d$ for $\chi$, which are independent from $P$.

Clearly, for $z \in \operatorname{supp}(\chi \theta)$, we have that $z \in B(y, r)$, for some $y \in p^{-1}(x)$, and in particular, $z$ is contained in a ball of the system. With respect to the corresponding coordinate system and trivialization, denoting by $\left(\theta^{(1)}, \ldots, \theta^{(\ell)}\right)$ the local expression of $\theta$, we have

$$
\begin{aligned}
\left\|D_{2}(\chi \theta)(z)\right\| & =\left\|\sum_{|\alpha| \leq d}\left(A^{\alpha} \circ p\right)(z) \frac{\partial^{|\alpha|}}{\partial y^{\alpha}}\left(\chi\left(\theta^{(1)}, \ldots, \theta^{(\ell)}\right)\right)(z)\right\| \\
& \leq \sum_{|\alpha| \leq d} C_{1}\left\|\frac{\partial^{|\alpha|}}{\partial y^{\alpha}}\left(\chi\left(\theta^{(1)}, \ldots, \theta^{(\ell)}\right)\right)(z)\right\| \\
& \leq C_{1} C_{2} C_{3} C(d, \ell),
\end{aligned}
$$

where $C_{3}$ is the uniform bound up to order $d$ for $\chi$ (which is independent from $P$ ) and $C(d, \ell)$ is a constant depending only on $d$ and $\ell$.

Corollary 4.6 There exists a constant $C^{\prime}$, independent from $P$, such that for any point $z \in M_{2}$, we have $\left|\left\langle D_{2}(\chi \theta)(z),(\chi \theta)(z)\right\rangle\right| \leq C^{\prime}$.

Proof Follows immediately from Proposition 4.5.

\subsection{Amenable Coverings}

In this subsection, we continue to work in the setting of the previous subsection; that is, we consider the Riemannian covering $p: M_{2} \rightarrow M_{1}$ and a fixed compact subset $K_{0}$ of $M_{1}$. Consider the universal coverings $p_{i}: \tilde{M} \rightarrow M_{i}$ and denote by $\Gamma_{i}$ the deck transformations group of $p_{i}, i=1,2$.

If $M_{1}$ has empty boundary, we consider a Riemannian metric $\mathfrak{h}_{1}$ conformal to the original metric $\mathfrak{g}_{1}$, such that $\left(M_{1}, \mathfrak{h}_{1}\right)$ is complete. Distances are considered with respect to $\mathfrak{h}_{1}$ or its lift $\mathfrak{h}_{2}$ on $M_{2}$. Similarly, the distance on $\tilde{M}$ is considered with respect to the lift of $\mathfrak{h}_{1}$. For $x \in M_{1}$ and $y \in p^{-1}(x)$, we denote by $D_{y}$ the fundamental domain of the Riemannian covering $p:\left(M_{2}, \mathfrak{h}_{2}\right) \rightarrow\left(M_{1}, \mathfrak{h}_{1}\right)$ centered at $y$.

If $M_{1}$ has non-empty boundary, we extend the Riemannian covering $p: M_{2} \rightarrow M_{1}$ to a Riemannian covering $p: N_{2} \rightarrow N_{1}$, according to Proposition 3.1. We consider a Riemannian metric $\mathfrak{h}_{1}$ on $N_{1}$ conformal to original Riemannian metric $\mathfrak{g}_{1}$, that coincides with $\mathfrak{g}_{1}$ in a compact neighborhood of $K_{0}$, such that $\left(N_{1}, \mathfrak{h}_{1}\right)$ is complete. From Lemma 3.3, $\tilde{M}$ can be identified with a domain of the simply connected covering space $\tilde{N}$ of $N_{1}$. Denote by $\mathfrak{h}_{2}$ and $\tilde{\mathfrak{h}}$ the lift of $\mathfrak{h}_{1}$ on $N_{2}$ and $\tilde{N}$, respectively. As distance function on $M_{1}, M_{2}$, and $\tilde{M}$, we consider the restriction of the distance function of $\left(N_{1}, \mathfrak{h}_{1}\right),\left(N_{2}, \mathfrak{h}_{2}\right)$, and $(\tilde{N}, \tilde{\mathfrak{h}})$, respectively. For $x \in M_{1}$ and $y \in p^{-1}(x)$, we denote 
by $D_{y}$ the part of the fundamental domain of $p:\left(N_{2}, \mathfrak{h}_{2}\right) \rightarrow\left(N_{1}, \mathfrak{h}_{1}\right)$ centered at $y$ that lies in $M_{2}$; that is,

$$
D_{y}=\left\{z \in M_{2}: d(z, y) \leq d\left(z, y^{\prime}\right) \text { for any } y^{\prime} \in p^{-1}(x)\right\}
$$

where $d(\cdot, \cdot)$ is the distance function of $N_{2}$ induced by $\mathfrak{h}_{2}$.

Fix $x \in M_{1}$ and $u \in p_{1}^{-1}(x)$. It is quite convenient to identify $\Gamma_{2} \backslash \Gamma_{1}$ with $p^{-1}(x)$, that is, $\Gamma_{2} \gamma$ is identified with $p_{2}(\gamma u)$, and study induced right action of $\Gamma_{1}$ on $p^{-1}(x)$. Clearly, if $y=p_{2}(\gamma u)$, for some $\gamma \in \Gamma_{1}$, then $y \cdot g=p_{2}(\gamma g u)$, for any $g \in \Gamma_{1}$. It is worth to point out that $p$ is amenable if and only if this right action of $\Gamma_{1}$ on $p^{-1}(x)$ is amenable.

For $r>0$, consider the finite set

$$
G_{r}:=\left\{g \in \Gamma_{1}: d(u, g u)<r\right\}
$$

and the subgroup $\left\langle G_{r}\right\rangle$ of $\Gamma_{1}$ generated by $G_{r}$. We are interested in the right action of $\left\langle G_{r}\right\rangle$ on $p^{-1}(x)$. The next remark is a simple description of the orbits of this action.

Remark 4.7 Let $y \in p^{-1}(x)$ and $g \in G_{r}$. Then there exists $\gamma \in \Gamma_{1}$, with $y=p_{2}(\gamma u)$ and $y \cdot g=p_{2}(\gamma g u)$. Clearly, we have

$$
d(y, y \cdot g)=d\left(p_{2}(\gamma u), p_{2}(\gamma g u)\right) \leq d(\gamma u, \gamma g u)=d(u, g u)<r .
$$

Conversely, let $y_{1}, y_{2} \in p^{-1}(x)$ with $d\left(y_{1}, y_{2}\right)<r$. Then there exist $\gamma_{1}, \gamma_{2} \in \Gamma_{1}$, such that $y_{i}=p_{2}\left(\gamma_{i} u\right)$, for $i=1,2$, and there exists $\sigma \in \Gamma_{2}$, such that

$$
d\left(\sigma \gamma_{1} u, \gamma_{2} u\right)=d\left(p_{2}\left(\gamma_{1} u\right), p_{2}\left(\gamma_{2} u\right)\right)=d\left(y_{1}, y_{2}\right)<r .
$$

This yields that $\gamma_{1}^{-1} \sigma^{-1} \gamma_{2}=: g \in G_{r}$. It follows that $\Gamma_{2} \gamma_{2}=\Gamma_{2} \gamma_{1} g$, i.e., $y_{2}=y_{1} \cdot g$.

Hence, two points $z_{1}, z_{2} \in p^{-1}(x)$ are in the same orbit of the action of $\left\langle G_{r}\right\rangle$ on $p^{-1}(x)$ if and only if there exist $k \in \mathbb{N}$ and $y_{1}, \ldots, y_{k} \in p^{-1}(x)$, such that $y_{1}=z_{1}$, $y_{k}=z_{2}$, and $d\left(y_{i}, y_{i+1}\right)<r$, for $i=1, \ldots, k-1$.

Lemma 4.8 If $p: M_{2} \rightarrow M_{1}$ is infinite sheeted, then there exists $R>0$, such that one of the following holds:

(i) either for any $r \geq R$, the action of $\left\langle G_{r}\right\rangle$ on $p^{-1}(x)$ has only infinite orbits,

(ii) or for any $r \geq R$, the action of $\left\langle G_{r}\right\rangle$ on $p^{-1}(x)$ has infinitely many finite orbits.

Proof Assume to the contrary that the statement does not hold. Then there exists $r_{0}>0$, such that the action of $\left\langle G_{r_{0}}\right\rangle$ on $p^{-1}(x)$ has only finitely many finite orbits $\mathcal{O}_{1}, \ldots, \mathcal{O}_{k}$, for some $k \in \mathbb{N}$. Since $p$ is infinite sheeted, there exists also an infinite orbit $\mathcal{O}$. Since the action of $\Gamma_{1}$ on $p^{-1}(x)$ is transitive, for $y_{i} \in \mathcal{O}_{i}$, there exists $g_{i} \in \Gamma_{1}$, such that $y_{i} \cdot g_{i} \in \mathcal{O}$, for $i=1, \ldots, k$. Then there exists $R>0$, such that $G_{r_{0}} \cup\left\{g_{1}, \ldots, g_{k}\right\} \subset G_{R}$ and the action of $\left\langle G_{R}\right\rangle$ on $p^{-1}(x)$ has only infinite orbits. It is clear that so does the action of $\left\langle G_{r}\right\rangle$ on $p^{-1}(x)$, for any $r \geq R$, which is a contradiction. 
Let $r>0$, such that $K_{0} \subset B(x, r)$. If $M_{1}$ has non-empty boundary, consider $r$ large enough, so that $B(u, r) \cap \partial \tilde{M} \neq \varnothing$. If $p$ is infinite sheeted, we choose $r \geq R$, where $R$ is the constant from Lemma 4.8. Consider a partition of unity consisting of the functions $\varphi_{1}$ and $\varphi_{y}$, with $y \in p^{-1}(x)$, associated with $K_{0}, x, u$, and $r$ as in (5). For a finite $P \subset p^{-1}(x)$, let $\chi:=\sum_{y \in P} \varphi_{y}$ and consider the sets

$$
\begin{aligned}
Q_{+} & :=\left\{y \in p^{-1}(x): \chi=1 \text { in } B(y, r)\right\} \\
Q_{-} & :=\left\{y \in p^{-1}(x): 0<\chi(z)<1 \text { for some } z \in B(y, r)\right\}, \\
Q & :=Q_{+} \cup Q_{-}=\left\{y \in p^{-1}(x): \chi(z) \neq 0 \text { for some } z \in B(y, r)\right\} .
\end{aligned}
$$

Clearly, $\chi=0$ in $B(y, r)$, for any $y \in p^{-1}(x) \backslash Q$. Since $\chi$ is compactly supported, it follows that $Q$ is finite. The proof of the following lemma is essentially presented in [2], but since we are in a different situation here, we repeat it.

Lemma 4.9 If $p$ is amenable, then for any $\varepsilon>0$, there exists a non-empty, finite $P \subset p^{-1}(x)$, such that

$$
\frac{\#\left(Q_{-}\right)}{\#\left(Q_{+}\right)}<\varepsilon
$$

Proof From Proposition 2.8, since $p$ is amenable, for any $\delta>0$, there exists a nonempty, finite $P \subset p^{-1}(x)$, such that

$$
\#(P \backslash P g)<\delta \#(P),
$$

for all $g \in G_{2 r+2}$. From Remark 4.3, we have that supp $\varphi_{y_{0}} \subset C\left(y_{0}, r+1\right), \varphi_{y_{0}}>0$ in $B\left(y_{0}, r+1 / 2\right)$, and $\sum_{y \in p^{-1}(x)} \varphi_{y}=1$ in $B\left(y_{0}, r\right)$, for any $y_{0} \in p^{-1}(x)$. Clearly, $P$ is contained in $Q$, which implies that $\#(P) \leq \#(Q)$.

For $y \in Q_{-}$, there exists $z \in B(y, r)$, such that $0<\chi(z)<1$. Therefore, there exist $y_{1} \in P$ and $y_{2} \in p^{-1}(x) \backslash P$, such that $\varphi_{y_{i}}(z)>0$, which yields that $d\left(y_{i}, z\right)<r+1$, for $i=1,2$. It follows that $d\left(y_{1}, y_{2}\right)<2 r+2$ and from Remark 4.7, there exists $g \in G_{2 r+2}$, such that $y_{1}=y_{2} \cdot g$. In particular, $y_{1} \in P \backslash P g$. Since $d\left(y, y_{1}\right)<2 r+1$, from Lemma 2.6, for a fixed $y_{1}$, there exist at most $N(2 r+1)$ such $y$. Since $y_{1} \in P \backslash P g$, for some $g \in G_{2 r+2}$, there exist at most $\delta \#(P) \#\left(G_{2 r+2}\right)$ such $y_{1}$. Hence, it follows that

$$
\#\left(Q_{-}\right) \leq \delta \#(P) \#\left(G_{2 r+2}\right) N(2 r+1) \leq \delta \#(Q) \#\left(G_{2 r+2}\right) N(2 r+1) .
$$

Since $Q$ is the disjoint union of $Q_{+}$and $Q_{-}$, for $\delta \#\left(G_{2 r+2}\right) N(2 r+1)<1$, we have

$$
\frac{\#\left(Q_{-}\right)}{\#\left(Q_{+}\right)} \leq \frac{\delta \#\left(G_{2 r+2}\right) N(2 r+1)}{1-\delta \#\left(G_{2 r+2}\right) N(2 r+1)} .
$$

This completes the proof, since $\delta>0$ is arbitrarily small. 
Proposition 4.10 If $p: M_{2} \rightarrow M_{1}$ is infinite sheeted and amenable, then for any $\varepsilon>0$ and $K \subset M_{2}$ compact, there exists a non-empty, finite $P \subset p^{-1}(x)$, such that $\operatorname{supp} \chi$ does not intersect $K$ and

$$
\frac{\#\left(Q_{-}\right)}{\#\left(Q_{+}\right)}<\varepsilon
$$

Proof First assume that the second statement of Lemma 4.8 holds. Then the action of $\left\langle G_{2 r+2}\right\rangle$ on $p^{-1}(x)$ has infinitely many finite orbits $\mathcal{O}_{n}$, with $n \in \mathbb{N}$. Clearly, for $P:=\mathcal{O}_{n}$, we have that $Q_{-}$is empty. Indeed, if there exists $y_{0} \in Q_{-}$, then there exist $z \in B\left(y_{0}, r\right), y_{1} \in P$, and $y_{2} \in p^{-1}(x) \backslash P$, such that $\varphi_{y_{i}}(z)>0, i=1,2$. It follows that $d\left(z, y_{i}\right)<r+1, i=1,2$, which yields that $d\left(y_{1}, y_{2}\right)<2 r+2$. From Remark 4.7, there exists $g \in G_{2 r+2}$, such that $y_{2}=y_{1} \cdot g$, which is a contradiction, since $P$ is an orbit of the action of $\left\langle G_{2 r+2}\right\rangle$ on $p^{-1}(x)$.

For a compact $K \subset M_{2}$, the set $P_{K}:=p^{-1}(x) \cap B(K, r+2)$ is finite and in particular, intersects only finitely many orbits $\mathcal{O}_{n}$. Let $P$ be an orbit that does not intersect $P_{K}$. Since supp $\varphi_{y} \subset C(y, r+1)$, for any $y \in p^{-1}(x)$, it is clear that for such $P$, the support of $\chi$ does not intersect $K$.

Assume now that the first statement of Lemma 4.8 holds, that is, the action of $\left\langle G_{r}\right\rangle$ on $p^{-1}(x)$ has only infinite orbits. For a compact subset $K$ of $M_{2}$, consider the finite set $P_{K}:=p^{-1}(x) \cap B(K, r+2)$. From Lemma 4.9, for any $\varepsilon>0$, there exists a non-empty, finite $P \subset p^{-1}(x)$, such that

$$
\frac{\#\left(Q_{-}\right)}{\#\left(Q_{+}\right)}<\delta:=\frac{\varepsilon}{1+(1+\varepsilon) N(2 r+1) \#\left(P_{K}\right)},
$$

where $N(2 r+1)$ is the constant from Lemma 2.6.

Since the action of $\left\langle G_{r}\right\rangle$ on $p^{-1}(x)$ has only infinite orbits, it follows that $Q_{-}$is non-empty. Indeed, since $P$ is non-empty and this action has only infinite orbits, there exists an infinite orbit $\mathcal{O}$ and $z_{1} \in P \cap \mathcal{O}$. Since $P$ is finite, there exists $z_{2} \in \mathcal{O} \backslash P$, and from Remark 4.7, there exist $k \in \mathbb{N}$ and $y_{1}, \ldots, y_{k} \in p^{-1}(x)$, with $y_{1}=z_{1}$, $y_{k}=z_{2}$, and $d\left(y_{i}, y_{i+1}\right)<r$, for $i=1, \ldots, k-1$. Since $y_{1} \in P$ and $y_{k} \notin P$, there exists $1 \leq j<k$, such that $y_{j} \in P$ and $y_{j+1} \notin P$. Since $d\left(y_{j}, y_{j+1}\right)<r$, it follows that $0<\chi\left(y_{j+1}\right)<1$ and in particular, $y_{j} \in Q_{-}$.

Evidently, $Q_{+}$is contained in $P$. Since $Q_{-}$is non-empty, it is clear that

$$
\frac{1}{\delta} \leq \#\left(Q_{+}\right) \leq \#(P)
$$

which yields that $\#(P)>\#\left(P_{K}\right)$, from the choice of $\delta$. In particular, the finite set $P^{\prime}:=P \backslash P_{K}$ is non-empty. Consider the function $\chi^{\prime}$ and the sets $Q_{+}^{\prime}, Q_{-}^{\prime}$, and $Q^{\prime}$ corresponding to $P^{\prime}$ as in (6). Clearly, the support of $\chi^{\prime}$ does not intersect $K$, since $\operatorname{supp} \varphi_{y} \subset C(y, r+1)$, for any $y \in p^{-1}(x)$. 
From Lemma 2.6, it follows that for any $y_{0} \in p^{-1}(x)$, the support of $\varphi_{y_{0}}$ intersects at most $N(2 r+1)$ open balls $B(y, r)$, with $y \in p^{-1}(x)$. Hence, we have that

$$
\begin{aligned}
& \#\left(Q_{-}^{\prime}\right) \leq \#\left(Q_{-}\right)+N(2 r+1) \#\left(P_{K}\right), \\
& \#\left(Q_{+}^{\prime}\right) \geq \#\left(Q_{+}\right)-N(2 r+1) \#\left(P_{K}\right) .
\end{aligned}
$$

Therefore, we obtain

$$
\frac{\#\left(Q_{-}^{\prime}\right)}{\#\left(Q_{+}^{\prime}\right)} \leq \frac{\#\left(Q_{-}\right)+N(2 r+1) \#\left(P_{K}\right)}{\#\left(Q_{+}\right)-N(2 r+1) \#\left(P_{K}\right)}<\varepsilon,
$$

from the choice of $\delta$.

Remark 4.11 After endowing $M_{1}$ or $N_{1}$ with $\mathfrak{h}_{1}$ (depending on whether $M_{1}$ has empty boundary or not) and the covering space with its lift $\mathfrak{h}_{2}$, we have that $p: D_{y} \rightarrow M_{1}$ is an isometry up to sets of measure zero, for any $y \in p^{-1}(x)$. Thus, for $f \in C_{c}\left(M_{1}\right)$, we have

$$
\int_{D_{y}}(f \circ p) d \mathrm{Vol}_{\mathfrak{h}_{2}}=\int_{M_{1}} f d \mathrm{Vol}_{\mathfrak{h}_{1}},
$$

where $\operatorname{Vol}_{\mathfrak{h}_{i}}$ (respectively, $\operatorname{Vol}_{\mathfrak{g}_{i}}$ ) is the volume element of $M_{i}$ induced by $\mathfrak{h}_{i}$ (respectively, $\left.\mathfrak{g}_{i}\right), i=1,2$. Since $\mathfrak{g}_{1}$ and $\mathfrak{h}_{1}$ are conformal, it is clear that there exists a positive $\varphi_{v} \in C^{\infty}\left(M_{1}\right)$, such that

$$
d \operatorname{Vol}_{\mathfrak{g}_{1}}=\varphi_{v} d \operatorname{Vol}_{\mathfrak{h}_{1}} \text { and } d \operatorname{Vol}_{\mathfrak{g}_{2}}=\left(\varphi_{v} \circ p\right) d \operatorname{Vol}_{\mathfrak{h}_{2}}
$$

In particular, for any $f \in C_{c}\left(M_{1}\right)$ and $y \in p^{-1}(x)$, from (7), we obtain that

$$
\begin{aligned}
\int_{D_{y}}(f \circ p) d \mu_{2} & =\int_{D_{y}}(f \circ p)(h \circ p)\left(\varphi_{v} \circ p\right) d \operatorname{Vol}_{\mathfrak{h}_{2}}=\int_{M_{1}} f h \varphi_{v} d \operatorname{Vol}_{\mathfrak{h}_{1}} \\
& =\int_{M_{1}} f d \mu_{1} .
\end{aligned}
$$

Similarly, for a compact $K \subset M_{1}$, we have $\mu_{1}(K)=\mu_{2}\left(p^{-1}(K) \cap D_{y}\right)$, for any $y \in p^{-1}(x)$.

Proposition 4.12 Let $p: M_{2} \rightarrow M_{1}$ be an infinite sheeted, amenable Riemannian covering. Let $\eta \in \mathcal{D}\left(D_{1}\right)$ with $\|\eta\|_{L^{2}\left(E_{1}, \mu_{1}\right)}=1$ and $\lambda \in \mathbb{F}$. Then for any $\varepsilon>0$ and $K \subset M_{2}$ compact, there exists $\zeta \in \mathcal{D}\left(D_{2}\right)$, with $\|\zeta\|_{L^{2}\left(E_{2}, \mu_{2}\right)}=1$, supp $\zeta \cap K=$ $\emptyset, \operatorname{supp} \zeta \subset p^{-1}(\operatorname{supp} \eta)$, and $\left\|\left(D_{2}-\lambda\right) \zeta\right\|_{L^{2}\left(E_{2}, \mu_{2}\right)} \leq\left\|\left(D_{1}-\lambda\right) \eta\right\|_{L^{2}\left(E_{1}, \mu_{1}\right)}+\varepsilon$.

Proof Let $K_{0}:=\operatorname{supp} \eta$. If $M_{1}$ has non-empty boundary, extend the Riemannian covering $p: M_{2} \rightarrow M_{1}$ according to Proposition 3.1. Consider conformal Riemannian metrics and distance functions as described in the beginning of this subsection. 
Let $p_{1}: \tilde{M} \rightarrow M_{1}$ be the universal covering of $M_{1}$ and fix $x \in M_{1}, u \in p_{1}^{-1}(x)$, and $r \geq R$ (from Lemma 4.8), such that $K_{0} \subset B(x, r)$. If $M_{1}$ has non-empty boundary, consider $r$ large enough, so that $B(u, r) \cap \partial \tilde{M} \neq \varnothing$. Consider a partition of unity consisting of the functions $\varphi_{1}$ and $\varphi_{y}$, with $y \in p^{-1}(x)$, associated with $K_{0}, x, u$, and $r$ as in (5), and let $\theta$ be the lift of $\eta$. From Remark 4.4, for any finite set $P^{\prime} \subset p^{-1}(x)$ and $\chi^{\prime}:=\sum_{y \in P^{\prime}} \varphi_{y}$, we have that $\chi^{\prime} \theta \in \mathcal{D}\left(D_{2}\right)$. From Proposition 4.5, there exists $C>0$, independent from $P^{\prime}$, such that $\left\|D_{2}\left(\chi^{\prime} \theta\right)(z)\right\| \leq C$, for any $z \in M_{2}$. Hence, we obtain that

$$
\max _{z \in M_{2}}\left\|\left(D_{2}-\lambda\right)\left(\chi^{\prime} \theta\right)(z)\right\| \leq C+|\lambda| \max _{w \in M_{1}}\|\eta(w)\|=: C_{0}
$$

From Proposition 4.10, there exists a non-empty, finite $P \subset p^{-1}(x)$, such that the support of $\chi:=\sum_{y \in P} \varphi_{y}$ does not intersect $K$ and

$$
\frac{\#\left(Q_{-}\right)}{\#\left(Q_{+}\right)}<\frac{\varepsilon}{C_{0}^{2} \mu_{1}(\operatorname{supp} \eta)}
$$

where $Q_{+}, Q_{-}$, and $Q$ are the sets corresponding to $P$ as in (6).

Since $\chi \theta$ is in the domain of $D_{2}$, so is the normalized section $\zeta:=\left(1 /\|\chi \theta\|_{L^{2}\left(E_{2}, \mu_{2}\right)}\right) \chi \theta$. Evidently, $\|\zeta\|_{L^{2}\left(E_{2}, \mu_{2}\right)}=1$ and $\operatorname{supp} \zeta \subset p^{-1}(\operatorname{supp} \eta)$. From Lemma 2.5, we have that $\operatorname{supp} \zeta \cap D_{y} \subset B(y, r)$, for any $y \in p^{-1}(x)$, which yields that $\operatorname{supp} \zeta$ is contained in the union of the fundamental domains $D_{y}$, with $y \in Q$. Clearly, we have

$$
\|\chi \theta\|_{L^{2}\left(E_{2}, \mu_{2}\right)}^{2} \geq \sum_{y \in Q_{+}} \int_{D_{y}}\|\chi \theta\|^{2} \mathrm{~d} \mu_{2}=\sum_{y \in Q_{+}} \int_{D_{y}}\|\theta\|^{2} \mathrm{~d} \mu_{2}=\#\left(Q_{+}\right),
$$

from the definition of $Q_{+}$and Remark 4.11. Therefore, we obtain that

$$
\begin{aligned}
\int_{M_{2}}\left\|\left(D_{2}-\lambda\right) \zeta\right\|^{2} \mathrm{~d} \mu_{2} \leq & \frac{1}{\#\left(Q_{+}\right)} \sum_{y \in Q_{+}} \int_{D_{y}}\left\|\left(D_{2}-\lambda\right)(\chi \theta)\right\|^{2} \mathrm{~d} \mu_{2} \\
& +\frac{1}{\#\left(Q_{+}\right)} \sum_{y \in Q_{-}} \int_{D_{y}}\left\|\left(D_{2}-\lambda\right)(\chi \theta)\right\|^{2} \mathrm{~d} \mu_{2}
\end{aligned}
$$

For $y \in Q_{+}$, we have $\chi=1$ in $B(y, r)$, which is a neighborhood of $\operatorname{supp} \theta \cap D_{y}$. This implies that

$$
\begin{aligned}
\frac{1}{\#\left(Q_{+}\right)} \sum_{y \in Q_{+}} \int_{D_{y}}\left\|\left(D_{2}-\lambda\right)(\chi \theta)\right\|^{2} \mathrm{~d} \mu_{2} & =\frac{1}{\#\left(Q_{+}\right)} \sum_{y \in Q_{+}} \int_{D_{y}}\left\|\left(D_{2}-\lambda\right) \theta\right\|^{2} \mathrm{~d} \mu_{2} \\
& =\int_{M_{1}}\left\|\left(D_{1}-\lambda\right) \eta\right\|^{2} \mathrm{~d} \mu_{1} .
\end{aligned}
$$


Since $\left\|\left(D_{2}-\lambda\right)(\chi \theta)(z)\right\| \leq C_{0}$, for any $z \in M_{2}$, it follows that

$$
\begin{aligned}
\frac{1}{\#\left(Q_{+}\right)} \sum_{y \in Q_{-}} \int_{D_{y}}\left\|\left(D_{2}-\lambda\right)(\chi \theta)\right\|^{2} \mathrm{~d} \mu_{2} & \leq \frac{C_{0}^{2}}{\#\left(Q_{+}\right)} \sum_{y \in Q_{-}} \mu_{2}\left(\operatorname{supp} \theta \cap D_{y}\right) \\
& =\frac{\#\left(Q_{-}\right)}{\#\left(Q_{+}\right)} C_{0}^{2} \mu_{1}(\operatorname{supp} \eta) \leq \varepsilon
\end{aligned}
$$

Hence, $\left\|\left(D_{2}-\lambda\right) \zeta\right\|_{L^{2}\left(E_{2}, \mu_{2}\right)}^{2} \leq\left\|\left(D_{1}-\lambda\right) \eta\right\|_{L^{2}\left(E_{1}, \mu_{1}\right)}^{2}+\varepsilon$.

Proposition 4.13 Let $p: M_{2} \rightarrow M_{1}$ be an infinite sheeted, amenable Riemannian covering, and assume that the operators $D_{i}$ are symmetric, $i=1,2$. Then for any section $\eta \in \mathcal{D}\left(D_{1}\right) \backslash\{0\}, \varepsilon>0$, and $K \subset M_{2}$ compact, there exists $\zeta \in \mathcal{D}\left(D_{2}\right) \backslash\{0\}$, such that $\operatorname{supp} \zeta \subset p^{-1}(\operatorname{supp} \eta)$, $\operatorname{supp} \zeta \cap K=\emptyset$, and $\mathcal{R}_{D_{2}}(\zeta) \leq \mathcal{R}_{D_{1}}(\eta)+\varepsilon$.

Proof The proof is similar to the proof of Proposition 4.12, using Corollary 4.6 instead of Proposition 4.5.

Proof of Theorem 4.1: Consider $\lambda \in \sigma_{\text {ap }}\left(\bar{D}_{1}\right)$. From Lemma 2.2, it follows that there exists $\left(\eta_{k}\right)_{k \in \mathbb{N}} \subset \mathcal{D}\left(D_{1}\right)$, such that $\left\|\eta_{k}\right\|_{L^{2}\left(E_{1}, \mu_{1}\right)}=1$ and $\left(D_{1}-\lambda\right) \eta_{k} \rightarrow 0$ in $L^{2}\left(E_{1}, \mu_{1}\right)$. Consider an exhausting sequence $\left(K_{k}\right)_{k \in \mathbb{N}}$ of $M_{2}$. From Proposition 4.12, for any $k \in \mathbb{N}$, there exists $\zeta_{k} \in \mathcal{D}\left(D_{2}\right)$, with $\left\|\zeta_{k}\right\|_{L^{2}\left(E_{2}, \mu_{2}\right)}=1$, such that $\|\left(D_{2}-\right.$ $\lambda) \zeta_{k}\left\|_{L^{2}\left(E_{2}, \mu_{2}\right)} \leq\right\|\left(D_{1}-\lambda\right) \eta_{k} \|_{L^{2}\left(E_{1}, \mu_{1}\right)}+1 / k$ and supp $\zeta_{k} \cap K_{k}=\emptyset$. Therefore, $\left(D_{2}-\lambda\right) \zeta_{k} \rightarrow 0$ in $L^{2}\left(E_{2}, \mu_{2}\right)$ and for any compact $K \subset M_{2}$, there exists $k_{0} \in \mathbb{N}$, such that $\operatorname{supp} \zeta_{k} \cap K=\emptyset$, for all $k \geq k_{0}$. It follows that $\left(\zeta_{k}\right)_{k \in \mathbb{N}}$ is a Weyl sequence for $D_{2}^{\prime}$ and $\lambda$, and in particular, $\lambda \in \sigma_{W}\left(D_{2}^{\prime}\right)$.

Proof of Theorem 1.1: Follows immediately from Theorem 4.1 and Proposition 2.1.

Assume now that the operator $D_{i}: \mathcal{D}\left(D_{i}\right) \subset L^{2}\left(E_{i}, \mu_{i}\right) \rightarrow L^{2}\left(E_{i}, \mu_{i}\right)$ is symmetric and bounded from below, and let $D_{i}^{(F)}$ be its Friedrichs extension, $i=1,2$. For more details on the Friedrichs extension of a symmetric, bounded from below and densely defined linear operator on a Hilbert space, see [25]. It is well known that the Friedrichs extension of an operator preserves its lower bound. In particular, for $i=1,2$, we have

$$
\lambda_{0}\left(D_{i}^{(F)}\right)=\inf _{\eta \in \mathcal{D}\left(D_{i}\right) \backslash\{0\}} \mathcal{R}_{D_{i}}(\eta)
$$

Recall the following proposition for the essential spectrum of a self-adjoint operator.

Proposition 4.14 ([13, Proposition 2.1]) Let $A: \mathcal{D}(A) \subset \mathcal{H} \rightarrow \mathcal{H}$ be a bounded from below, self-adjoint operator on a separable Hilbert space $\mathcal{H}$ over $\mathbb{R}$ or $\mathbb{C}$, and consider $\lambda \in \mathbb{R}$. Then the interval $(-\infty, \lambda]$ intersects the essential spectrum of $A$ if and only iffor any $\varepsilon>0$, there exists an infinite-dimensional subspace $\mathcal{H}_{\varepsilon} \subset \mathcal{D}(A)$, such that $\mathcal{R}_{A}(v)<\lambda+\varepsilon$, for all $v \in \mathcal{H}_{\varepsilon} \backslash\{0\}$. 
Proof of Theorem 1.2: From (8), it follows that there exists $\left(\eta_{k}\right)_{k \in \mathbb{N}} \subset \mathcal{D}\left(D_{1}\right) \backslash\{0\}$, such that $\mathcal{R}_{D_{1}}\left(\eta_{k}\right) \leq \lambda_{0}\left(D_{1}^{(F)}\right)+1 / k$, for any $k \in \mathbb{N}$. Proposition 4.13 yields that there exists $\left(\zeta_{k}\right)_{k \in \mathbb{N}} \subset \mathcal{D}\left(D_{2}\right) \backslash\{0\}$, such that $\mathcal{R}_{D_{2}}\left(\zeta_{k}\right) \leq \lambda_{0}\left(D_{1}^{(F)}\right)+2 / k$ and $\operatorname{supp} \zeta_{k} \cap \operatorname{supp} \zeta_{k^{\prime}}=\emptyset$, for all $k, k^{\prime} \in \mathbb{N}$, with $k \neq k^{\prime}$. Evidently, for any $\varepsilon>0$, there exists $k_{0} \in \mathbb{N}$, such that $\mathcal{R}_{D_{2}}\left(\zeta_{k}\right)<\lambda_{0}\left(D_{1}^{(F)}\right)+\varepsilon$, for all $k \geq k_{0}$. Consider the subspace $\mathcal{H}_{\varepsilon}$ of $\mathcal{D}\left(D_{2}\right)$ spanned by $\left\{\zeta_{k}: k \geq k_{0}\right\}$. Since the sections $\zeta_{k}$, with $k \in \mathbb{N}$, have disjoint supports, the space $\mathcal{H}_{\varepsilon}$ is infinite-dimensional. Clearly, any $\theta \in \mathcal{H}_{\varepsilon}$ is of the form $\theta:=\sum_{i=k_{0}}^{k_{0}+k} m_{i} \zeta_{i}$, for some $k \in \mathbb{N}$ and $m_{k_{0}}, \ldots, m_{k_{0}+k} \in \mathbb{F}$. Therefore, we have

$$
\mathcal{R}_{D_{2}}(\theta)=\frac{\sum_{i=k_{0}}^{k_{0}+k}\left|m_{i}\right|^{2}\left\langle D_{2} \zeta_{i}, \zeta_{i}\right\rangle_{L^{2}\left(E_{2}, \mu_{2}\right)}}{\sum_{i=k_{0}}^{k_{0}+k}\left|m_{i}\right|^{2}\left\|\zeta_{i}\right\|_{L^{2}\left(E_{2}, \mu_{2}\right)}^{2}} \leq \max _{k_{0} \leq i \leq k_{0}+k} \mathcal{R}_{D_{2}}\left(\zeta_{i}\right)<\lambda_{0}\left(D_{1}^{(F)}\right)+\varepsilon
$$

From Proposition 4.14, it follows that $\lambda_{0}^{\text {ess }}\left(D_{2}^{(F)}\right) \leq \lambda_{0}\left(D_{1}^{(F)}\right)$.

Remark 4.15 In the proof of Theorem 1.2, the only properties of the Friedrichs extension used are self-adjointness and the preservation of the lower bound of $D_{1}$. Therefore, this proof establishes the analogous result for any self-adjoint extensions of the operators, as long as the extension of $D_{1}$ preserves its lower bound.

The next proposition provides the analogous result in case the operators are symmetric and $D_{1}$ is not bounded from below.

Proposition 4.16 Assume that the operator $D_{i}$ is symmetric, $i=1,2$. If the covering is infinite sheeted and amenable, and $D_{1}$ is not bounded from below, then $D_{2}$ is not bounded from below.

Proof Since $D_{1}$ is not bounded from below, for any $C \in \mathbb{R}$, there exists a nonzero $\eta \in \mathcal{D}\left(D_{1}\right)$, with $\mathcal{R}_{D_{1}}(\eta) \leq C$. From Proposition 4.13, it follows that there exists $\zeta \in \mathcal{D}\left(D_{2}\right) \backslash\{0\}$, with $\mathcal{R}_{D_{2}}(\zeta)<C+1$. Therefore, $D_{2}$ is not bounded from below.

For sake of completeness, we also present the analogous results for finite-sheeted coverings. It is clear that they cannot be improved in order to obtain as strong statements as in the case of infinite- sheeted amenable coverings.

Proposition 4.17 Let $D_{2}^{\prime}$ be a closed extension of $D_{2}$. If $p$ is a finite-sheeted Riemannian covering, then $\sigma_{\mathrm{ap}}\left(\bar{D}_{1}\right) \subset \sigma_{\mathrm{ap}}\left(D_{2}^{\prime}\right)$ and $\sigma_{W}\left(\bar{D}_{1}\right) \subset \sigma_{W}\left(D_{2}^{\prime}\right)$.

Proof If $\eta$ is in the domain of $D_{1}$, then its lift is in the domain of $D_{2}$. For $\lambda \in \sigma_{W}\left(\bar{D}_{1}\right)$, from Lemma 2.2, there exists a Weyl sequence $\left(\eta_{k}\right)_{k \in \mathbb{N}} \subset \mathcal{D}\left(D_{1}\right)$ for $\bar{D}_{1}$ and $\lambda$. Then, the sequence consisting of the normalized (in $L^{2}\left(E_{2}, \mu_{2}\right)$ ) lifts of $\eta_{k}, k \in \mathbb{N}$, is a Weyl sequence for $D_{2}^{\prime}$ and $\lambda$. Hence, $\sigma_{W}\left(\bar{D}_{1}\right) \subset \sigma_{W}\left(D_{2}^{\prime}\right)$. Similarly, it follows that $\sigma_{\text {ap }}\left(\bar{D}_{1}\right) \subset \sigma_{\text {ap }}\left(D_{2}^{\prime}\right)$.

Proposition 4.18 Assume that $D_{i}$ is symmetric and bounded from below, and denote by $D_{i}^{(F)}$ its Friedrichs extension, $i=1$, 2. If $p$ is a finite-sheeted Riemannian covering, then $\lambda_{0}\left(D_{2}^{(F)}\right) \leq \lambda_{0}\left(D_{1}^{(F)}\right)$. 
Proof If $\eta$ is in the domain of $D_{1}$, then its lift $\theta$ is in the domain of $D_{2}$. If $\eta \neq 0$, it is easy to see that $\mathcal{R}_{D_{1}}(\eta)=\mathcal{R}_{D_{2}}(\theta)$, and the statement follows from (8).

In the rest of this section, we give applications of our results in the case of Schrödinger operators. Recall that on manifolds with boundary, we are interested in the Dirichlet spectrum of Schrödinger operators. The following proposition characterizes the bottom of the spectrum of a Schrödinger operator as the maximum of its positive spectrum.

Proposition 4.19 Let $S$ be a Schrödinger operator on a Riemannian manifold M. Then the bottom of the spectrum of $S$ is the maximum of all $\lambda \in \mathbb{R}$, such that there exists $\varphi \in C^{\infty}(M \backslash \partial M)$ with $S \varphi=\lambda \varphi$, which is positive in $M \backslash \partial M$.

Proof If $M$ has empty boundary, then the statement may be found in [11, Theorem 7], [14, Theorem 1], and [23, Theorem 2.1]. If $M$ has non-empty boundary, it is clear that $\lambda_{0}(S)=\lambda_{0}(S, M \backslash \partial M)$, where $\lambda_{0}(S, M \backslash \partial M)$ stands for the bottom of the spectrum of $S$ on the interior of $M$. Hence, in this case, the claim follows from the corresponding statement for manifolds without boundary.

In particular, there exists $\varphi \in C^{\infty}(M \backslash \partial M)$ with $S \varphi=\lambda_{0}(S) \varphi$, which is positive in the interior of $M$. It is worth to point out that the smooth eigenfunctions of the preceding proposition do not have to be square-integrable. The following corollary is a consequence of Proposition 4.19 (an alternative proof can be found in [2]).

Corollary 4.20 Let $p: M_{2} \rightarrow M_{1}$ be a Riemannian covering. Let $S_{1}$ be a Schrödinger operator on $M_{1}$ and $S_{2}$ its lift on $M_{2}$. Then $\lambda_{0}\left(S_{1}\right) \leq \lambda_{0}\left(S_{2}\right)$.

Proof Follows immediately from Proposition 4.19, since the lift of an eigenfunction of $S_{1}$ is an eigenfunction of $S_{2}$.

Corollary 4.21 Let $p: M_{2} \rightarrow M_{1}$ be an infinite-sheeted, amenable Riemannian covering. Let $S_{1}$ be a Schrödinger operator on $M_{1}$ and $S_{2}$ its lift on $M_{2}$. Then $\lambda_{0}\left(S_{1}\right)=\lambda_{0}^{\mathrm{ess}}\left(S_{2}\right)$. If, in addition, $M_{1}$ is complete, then $\sigma\left(S_{1}\right) \subset \sigma_{\mathrm{ess}}\left(S_{2}\right)$.

Proof Follows from Theorems 1.1, 1.2 and Corollary 4.20.

The following results describe the behavior of the spectrum of Schrödinger operators under finite-sheeted coverings.

Corollary 4.22 Let $p: M_{2} \rightarrow M_{1}$ be a finite-sheeted Riemannian covering. Let $S_{1}$ be a Schrödinger operator on $M_{1}$ and $S_{2}$ its lift on $M_{2}$. Then $\lambda_{0}\left(S_{1}\right)=\lambda_{0}\left(S_{2}\right)$. If, in addition, $M_{1}$ is complete, then $\sigma\left(S_{1}\right) \subset \sigma\left(S_{2}\right)$ and $\sigma_{\mathrm{ess}}\left(S_{1}\right) \subset \sigma_{\mathrm{ess}}\left(S_{2}\right)$.

Proof Follows from Propositions 2.1, 4.17, 4.18 and Corollary 4.20.

The following characterization of the bottom of the essential spectrum of a Schrödinger operator follows from the Decomposition Principle ([3, Proposition 1]) and Propositions 2.3 and 4.14. Recall that this quantity is infinite when the spectrum is discrete. 
Proposition 4.23 ([5, Proposition 3.2]) Let S be a Schrödinger operator on a complete manifold $M$ and let $\left(K_{k}\right)_{k \in \mathbb{N}}$ be an exhausting sequence of $M$. Then

$$
\lambda_{0}^{\mathrm{ess}}(S)=\lim _{k} \lambda_{0}\left(S, M \backslash K_{k}\right),
$$

where $\lambda_{0}\left(S, M \backslash K_{k}\right)$ is the bottom of the spectrum of $S$ on $M \backslash K_{k}$.

Corollary 4.24 Let $p: M_{2} \rightarrow M_{1}$ be a finite-sheeted Riemannian covering of complete manifolds. Consider a Schrödinger operator $S_{1}$ on $M_{1}$ and its lift $S_{2}$ on $M_{2}$. Then $\lambda_{0}^{\mathrm{ess}}\left(S_{1}\right)=\lambda_{0}^{\mathrm{ess}}\left(S_{2}\right)$ and in particular, $\sigma_{\mathrm{ess}}\left(S_{1}\right) \neq \emptyset$ if and only if $\sigma_{\mathrm{ess}}\left(S_{2}\right) \neq \emptyset$.

Proof Follows from Corollary 4.22 and Proposition 4.23.

\section{Infinite Deck Transformations Group}

Let $M$ be a Riemannian manifold, $E \rightarrow M$ a Riemannian or Hermitian vector bundle, endowed with a connection $\nabla$ and $D: \Gamma(E) \rightarrow \Gamma(E)$ a differential operator on $E$.

If $M$ has empty boundary, set $\mathcal{D}(D):=\Gamma_{c}(E)$. If $M$ has non-empty boundary, consider $v \in \mathbb{N}$, and for $l=1, \ldots, v$, let $k_{l} \in \mathbb{N}$ and $a_{j, l}$ be real or complex-valued functions (depending on whether $E$ is Riemannian or Hermitian) defined on $\partial M$, $j=0, \ldots, k_{l}$. It is worth to point out that we do not impose any assumptions on the functions $a_{j, l}$. Let $n$ be the inward pointing normal to $\partial M$ and consider

$$
\mathcal{D}(D):=\left\{\eta \in \Gamma_{c}(E): \sum_{j=0}^{k_{l}} a_{j, l} \nabla_{n}^{(j)} \eta=0 \text { on } \partial M, l=1, \ldots, \nu\right\} .
$$

Let $\mu$ be a measure on $M$ expressed via a positive smooth density with respect to the volume element of $M$; that is, there exists a positive $h \in C^{\infty}(M)$, such that $d \mu=h d$ Vol. Consider $D$ as a densely defined operator

$$
D: \mathcal{D}(D) \subset L^{2}(M, \mu) \rightarrow L^{2}(M, \mu) .
$$

When we refer to closability or symmetry of $D$, we consider it as in (9). From Lemma 2.4, the operator $D$ is closable and denote by $\bar{D}$ its closure.

Theorem 5.1 Let $\Gamma$ be a group of automorphisms of $E$ preserving the metric of $E$, such that the induced action on $M$ is isometric and $D\left(g_{*} \eta\right)=g_{*} D \eta$, for any $g \in \Gamma$ and $\eta \in \Gamma(E)$. Moreover, assume that the density function $h$ of $\mu$ is $\Gamma$-invariant. If $M$ has non-empty boundary, assume that $\nabla$ and the functions $a_{j . l}$ are $\Gamma$-invariant along the boundary. If for any compact $K \subset M$, there exists $g \in \Gamma$, such that $g K \cap K=\emptyset$, then $\sigma_{\mathrm{ap}}(\bar{D})=\sigma_{W}(\bar{D})$ and $\bar{D}$ does not have eigenvalues of finite multiplicity.

Proof Let $\lambda \in \sigma_{\mathrm{ap}}(\bar{D})$. From Lemma 2.2, there exists $\left(\eta_{k}\right)_{k \in \mathbb{N}} \subset \mathcal{D}(D)$, such that $\left\|\eta_{k}\right\|_{L^{2}(E, \mu)}=1$ and $(D-\lambda) \eta_{k} \rightarrow 0$ in $L^{2}(E, \mu)$. Since $\eta_{k}$ is compactly supported, there exists an exhausting sequence $\left(K_{k}\right)_{k \in \mathbb{N}}$ of $M$, such that supp $\eta_{k} \subset K_{k}$, for 
all $k \in \mathbb{N}$. For any $k \in \mathbb{N}$, consider $g_{k} \in \Gamma$, such that $g_{k} K_{k} \cap K_{k}=\emptyset$, and set $\zeta_{k}:=\left(g_{k}\right)_{\star} \eta_{k}$. Then $\zeta_{k} \in \Gamma_{c}(E)$ and if $M$ has non-empty boundary, then $\zeta_{k}$ satisfies the same boundary conditions with $\eta_{k}$, since via isometries unit speed geodesics normal to the boundary are mapped to unit speed geodesics normal to the boundary. It follows that $\zeta_{k} \in \mathcal{D}(D),\left\|\zeta_{k}\right\|_{L^{2}(E, \mu)}=1$, and $(D-\lambda) \zeta_{k} \rightarrow 0$ in $L^{2}(E, \mu)$. It is clear that $\operatorname{supp} \zeta_{k}=g_{k}\left(\operatorname{supp} \eta_{k}\right)$, which yields that for any compact $K \subset M$, there exists $k_{0} \in \mathbb{N}$, such that $\operatorname{supp} \zeta_{k} \cap K=\emptyset$, for all $k \geq k_{0}$. This implies that $\zeta_{k} \rightarrow 0$ in $L^{2}(E, \mu)$, that is, $\left(\zeta_{k}\right)_{k \in \mathbb{N}}$ is a Weyl sequence for $\bar{D}$ and $\lambda$. Hence, $\lambda \in \sigma_{W}(\bar{D})$.

Assume that there exists an eigenvalue $\lambda$ of $\bar{D}$ of finite multiplicity, and consider $\theta \in \mathcal{D}(\bar{D})$ with $\|\theta\|_{L^{2}(E, \mu)}=1$ and $\bar{D} \theta=\lambda \theta$. Then there exists $\left(\eta_{k}\right)_{k \in \mathbb{N}} \subset \mathcal{D}(D)$, such that $\eta_{k} \rightarrow \theta$ and $D \eta_{k} \rightarrow \bar{D} \theta$. It is clear that for any $g \in \Gamma$ and $k \in \mathbb{N}$, we have $g_{*} \eta_{k} \in \mathcal{D}(D), g_{*} \eta_{k} \rightarrow g_{*} \theta$, and $D\left(g_{*} \eta_{k}\right) \rightarrow g_{*}(\bar{D} \theta)$, which yields that $g_{*} \theta \in \mathcal{D}(\bar{D})$ and $\bar{D}\left(g_{*} \theta\right)=\lambda\left(g_{*} \theta\right)$.

Let $\left(K_{k}\right)_{k \in \mathbb{N}}$ be an exhausting sequence of $M$ and consider $\left(g_{k}\right)_{k \in \mathbb{N}} \subset \Gamma$, such that $g_{k} K_{k} \cap K_{k}=\emptyset$, for any $k \in \mathbb{N}$. It is clear that the sections $\theta_{k}:=\left(g_{k}\right)_{*} \theta$ satisfy $\bar{D} \theta_{k}=\lambda \theta_{k}$ and $\left\|\theta_{k}\right\|_{L^{2}(E, \mu)}=1$, for all $k \in \mathbb{N}$. Since the eigenspace corresponding to $\lambda$ is finite-dimensional, after passing to a subsequence, we may assume that $\theta_{k} \rightarrow \theta_{0}$ in $L^{2}(E, \mu)$, for some $\theta_{0}$, with $\left\|\theta_{0}\right\|_{L^{2}(E, \mu)}=1$. Consider a non-zero $\zeta \in \Gamma_{c}(E)$ and set $\zeta_{k}:=\left(g_{k}^{-1}\right)_{*} \zeta$. Then

$$
\left\langle\theta_{k}, \zeta\right\rangle_{L^{2}(E, \mu)}^{2}=\left\langle\theta, \zeta_{k}\right\rangle_{L^{2}(E, \mu)}^{2} \leq\|\zeta\|_{L^{2}(E, \mu)}^{2} \int_{\operatorname{supp} \zeta_{k}}\|\theta\|^{2} \mathrm{~d} \mu .
$$

Let $\varepsilon>0$ and consider a compact $K \subset M$, such that $\int_{M \backslash K}\|\theta\|^{2} \mathrm{~d} \mu<\varepsilon^{2} /\|\zeta\|_{L^{2}(E, \mu)}^{2}$. Since $\operatorname{supp} \zeta$ and $K$ are eventually subsets of $K_{k}$, there exists $k_{0} \in \mathbb{N}$, such that $\operatorname{supp} \zeta_{k} \cap K=\emptyset$, for all $k \geq k_{0}$. Therefore, for $k \geq k_{0}$, we have $\operatorname{supp} \zeta_{k} \subset M \backslash K$, and in particular, $\left|\left\langle\theta_{k}, \zeta\right\rangle_{L^{2}(E, \mu)}\right|<\varepsilon$. This yields that $\theta_{k} \rightarrow 0$ in $L^{2}(E, \mu)$, which is a contradiction, since $\theta_{k} \rightarrow \theta_{0}$ in $L^{2}(E, \mu)$ and $\left\|\theta_{0}\right\|_{L^{2}(E, \mu)}=1$.

Theorem 5.2 Assume that $D$ is symmetric and bounded from below, and denote by $D^{(F)}$ its Friedrichs extension. Under the assumptions of Theorem 5.1, the spectrum of $D^{(F)}$ is essential and $D^{(F)}$ does not have eigenvalues of finite multiplicity.

Proof Let $\eta \in \mathcal{D}\left(D^{(F)}\right)$ and $g \in \Gamma$. From the invariance of $\mathcal{D}(D)$ and $D$ under the action of $\Gamma$, it follows that $g_{*} \eta \in \mathcal{D}\left(D^{(F)}\right)$ and $D^{(F)}\left(g_{*} \eta\right)=g_{*}\left(D^{(F)} \eta\right)$. As in the proof of Theorem 5.1, it follows that $D^{(F)}$ does not have eigenvalues of finite multiplicity. From Proposition 2.1, we obtain that $\sigma\left(D^{(F)}\right)=\sigma_{\mathrm{ess}}\left(D^{(F)}\right)$.

The above theorems can be applied to Riemannian coverings with infinite deck transformations group. In the context of the previous section, we obtain the following consequences.

Corollary 5.3 If the deck transformations group of the covering is infinite, then $\bar{D}_{2}$ does not have eigenvalues of finite multiplicity and $\sigma_{\mathrm{ap}}\left(\bar{D}_{2}\right)=\sigma_{W}\left(\bar{D}_{2}\right)$.

Proof Follows immediately from Theorem 5.1, for $\Gamma$ being the deck transformations group of the covering. 
Proof of Corollary 1.3: Follows from Corollary 5.3 and Proposition 2.1.

Proof of Corollary 1.4: Follows from Theorem 5.2, for $\Gamma$ being the deck transformations group of the covering.

Corollary 5.4 Let $\Gamma$ be an infinite, discrete group acting properly discontinuously on a complete Riemannian manifold $M$ via isometries. Then there exists no non-zero, square-integrable, $\lambda_{0}(M)$-harmonic function on M. Moreover, $\lambda_{0}(M)$ is an accumulation point of $\sigma(M)$.

Proof For any complete (and connected) Riemannian manifold $M$, the space of squareintegrable, $\lambda_{0}(M)$-harmonic functions is either trivial or one-dimensional. Therefore, Corollary 1.3 yields the first statement and that $\lambda_{0}(M) \in \sigma_{\text {ess }}(M)$. The second statement follows from Proposition 2.1, since $\sigma_{\text {ess }}(M)$ consists of eigenvalues of infinite multiplicity and accumulation points of the spectrum.

Besides Riemannian coverings, the above theorems can be applied to manifolds with high symmetry. For instance, it follows that the spectrum of the Laplacian on a non-compact homogeneous space is essential. Moreover, we obtain the analogous statement, if there exists a non-compact Lie group acting on the manifold properly discontinuously via isometries.

\section{Coverings of Closed Manifolds}

The Cheeger's constant of a Riemannian manifold $M$ is defined by

$$
h(M):=\inf _{K} \frac{\operatorname{Area}(\partial K)}{\operatorname{Vol}(K)}
$$

where the infimum is taken over all compact and smoothly bounded domains $K$ of $M$ which do not intersect $\partial M$. It is related to $\lambda_{0}(M)$ via Cheeger's inequality (cf. [10]):

$$
\lambda_{0}(M) \geq \frac{1}{4} h(M)^{2} .
$$

Brooks [6] actually proved that a normal Riemannian covering of a closed manifold is amenable if and only if the Cheeger's constant of the covering space is zero. The following result is an extension of that of Brooks, to not necessarily normal coverings.

Theorem 6.1 Let $p: M_{2} \rightarrow M_{1}$ be a Riemannian covering of a closed manifold $M_{1}$. If $h\left(M_{2}\right)=0$, then $p$ is amenable.

In order to prove this theorem, we need the following proposition. In the sequel, for a subset $W$ of $M$, we denote by $B(W, r)$ the tubular neighborhood

$$
B(W, r):=\{z \in M: d(z, W)<r\}
$$


Proposition 6.2 ([9, Lemma 7.2]) Let $M$ be a non-compact, complete Riemannian manifold, without boundary and with Ricci curvature bounded from below. Then there exists a constant $c$ depending only on the dimension of $M$, such that for any compact and smoothly bounded domain $K$ of $M$, with $\operatorname{Area}(\partial K) / \operatorname{Vol}(K)=: H$, and any $0<r \leq 1 / 2 c \min \{1,1 / H\}$, there exists a bounded, open $U \subset M$, such that

$$
\frac{\operatorname{Vol}(B(\partial U, r))}{\operatorname{Vol}(U)} \leq C(r) H
$$

where $C(r)$ is a constant depending on $r$, the dimension of $M$, and the lower bound of the Ricci curvature.

Corollary 6.3 Let $M$ be a non-compact, complete Riemannian manifold, without boundary and with Ricci curvature bounded from below. If $h(M)=0$, then for any $\varepsilon, r>0$, there exists a bounded, open $U \subset M$, such that

$$
\frac{\operatorname{Vol}(B(\partial U, r))}{\operatorname{Vol}(U \backslash B(\partial U, r))}<\varepsilon
$$

Proof Let $r>0$ and $0<r_{0} \leq 1 / 2 c$, where $c$ is the constant from Proposition 6.2. Denote by $\mathfrak{g}$ the original Riemannian metric and consider the metric $\mathfrak{h}:=C \mathfrak{g}$, where $C:=r_{0} / r$. For any compact and smoothly bounded domain $K$ of $M$, we have

$$
\frac{\operatorname{Area}_{\mathfrak{h}}(\partial K)}{\operatorname{Vol}_{\mathfrak{h}}(K)}=C^{-1 / 2} \frac{\operatorname{Area}_{\mathfrak{g}}(\partial K)}{\operatorname{Vol}_{\mathfrak{g}}(K)} .
$$

Since the Cheeger's constant of $M$ with respect to $\mathfrak{g}$ is zero, it follows that so is the Cheeger's constant of $M$ with respect to $\mathfrak{h}$. From Proposition 6.2, for any $\delta>0$, there exists a bounded, open $U \subset M$, such that

$$
\frac{\operatorname{Vol}_{\mathfrak{h}}\left(B_{\mathfrak{h}}\left(\partial U, r_{0}\right)\right)}{\operatorname{Vol}_{\mathfrak{h}}(U)}<\delta
$$

It follows that

$$
\frac{\operatorname{Vol}_{\mathfrak{g}}\left(B_{\mathfrak{g}}(\partial U, r)\right)}{\operatorname{Vol}_{\mathfrak{g}}(U)}=\frac{\operatorname{Vol}_{\mathfrak{h}}\left(B_{\mathfrak{g}}(\partial U, r)\right)}{\operatorname{Vol}_{\mathfrak{h}}(U)}=\frac{\operatorname{Vol}_{\mathfrak{h}}\left(B_{\mathfrak{h}}\left(\partial U, r_{0}\right)\right)}{\operatorname{Vol}_{\mathfrak{h}}(U)}<\delta
$$

This completes the proof, since $\operatorname{Vol}_{\mathfrak{g}}(U) \leq \operatorname{Vol}_{\mathfrak{g}}\left(U \backslash B_{\mathfrak{g}}(\partial U, r)\right)+\operatorname{Vol}_{\mathfrak{g}}\left(B_{\mathfrak{g}}(\partial U, r)\right)$.

Proof of Theorem 6.1: Evidently, if $M_{2}$ is closed, then $p$ is finite sheeted and in particular, amenable. Therefore, it remains to prove the statement for $M_{2}$ non-compact. Consider the universal covering $p_{1}: \tilde{M} \rightarrow M_{1}$, fix $x \in M_{1}, u \in p_{1}^{-1}(x)$, and identify $\pi_{1}\left(M_{2}\right) \backslash \pi_{1}\left(M_{1}\right)$ with $p^{-1}(x)$, as in the beginning of Subsect. 4.2. Denote by $D_{y}$ 
the fundamental domain of $p$ centered at $y$, with $y \in p^{-1}(x)$. It is clear that for $y \in p^{-1}(x)$ and $z, w \in D_{y}$, we have

$$
d(z, w) \leq d(y, z)+d(y, w)=d(x, p(z))+d(x, p(w)) \leq 2 \operatorname{diam}\left(M_{1}\right),
$$

which yields that $\operatorname{diam}\left(D_{y}\right) \leq 2 \operatorname{diam}\left(M_{1}\right)$, for all $y \in p^{-1}(x)$. Let $r>2 \operatorname{diam}\left(M_{1}\right)$ and

$$
G_{r}:=\left\{g \in \pi_{1}\left(M_{1}\right): d(u, g u)<r\right\} .
$$

From Corollary 6.3, for any $\varepsilon>0$, there exists a bounded, open $U \subset M_{2}$, such that

$$
\frac{\operatorname{Vol}(B(\partial U, 2 r))}{\operatorname{Vol}(U \backslash B(\partial U, 2 r))}<\varepsilon
$$

Consider the finite sets

$$
\begin{aligned}
F & :=\left\{y \in p^{-1}(x): y \in U \backslash B(\partial U, r)\right\}, \\
F^{\prime} & :=\left\{y \in p^{-1}(x): y \in B(\partial U, r)\right\} .
\end{aligned}
$$

Recall that $r>2 \operatorname{diam}\left(M_{1}\right) \geq \operatorname{diam}\left(D_{y}\right)$, for all $y \in p^{-1}(x)$, and $M_{2}$ is covered by the fundamental domains $D_{y}$, with $y \in p^{-1}(x)$. Evidently, $U \backslash B(\partial U, 2 r)$ is contained in the union of $D_{y}$, with $y \in F$. Furthermore, $B(\partial U, 2 r)$ contains the union of $D_{y}$, with $y \in F^{\prime}$. From (10), since the intersection of different fundamental domains is of measure zero, and $\operatorname{Vol}\left(D_{y}\right)=\operatorname{Vol}\left(M_{1}\right)$, for any $y \in p^{-1}(x)$, it follows that

$$
\frac{\#\left(F^{\prime}\right)}{\#(F)}<\varepsilon .
$$

Let $g \in G_{r}$ and $y \in F \backslash F g$. Then $y \in U, d(y, \partial U) \geq r$ and $y \cdot g^{-1} \notin F$. From Remark 4.7, it follows that $d\left(y, y \cdot g^{-1}\right)<r$. Therefore, $y \cdot g^{-1} \in U$ and $d\left(y \cdot g^{-1}, \partial U\right)<r$, which yields that $y \cdot g^{-1} \in F^{\prime}$. Hence, $F \backslash F g \subset F^{\prime} g$ and in particular, we obtain that

$$
\#(F \backslash F g) \leq \#\left(F^{\prime}\right)<\varepsilon \#(F) .
$$

For any finite $G \subset \pi_{1}\left(M_{1}\right)$, there exists $r>2 \operatorname{diam}\left(M_{1}\right)$, such that $G \subset G_{r}$. The above arguments imply that for any finite $G \subset \pi_{1}\left(M_{1}\right)$ and $\varepsilon>0$, there exists a Følner set for $G$ and $\varepsilon$. From Proposition 2.8, it follows that $p$ is amenable.

\section{Applications and Examples}

We begin with some examples of operators for which our main results can be applied. 
Examples 7.1 In the following examples, we consider a Riemannian manifold $M$. If $M$ has non-empty boundary, we denote by $n$ the inward pointing normal to $\partial M$.

(i) Schrödinger operators. A Schrödinger operator on $M$ is an operator of the form $S=\Delta+V$, where $\Delta$ is the Laplacian and $V \in C^{\infty}(M)$ is bounded from below. If $M$ has empty boundary, then the operator

$$
S: C_{c}^{\infty}(M) \subset L^{2}(M) \rightarrow L^{2}(M)
$$

is symmetric and bounded from below. If, in addition, $M$ is complete, then this operator is essentially self-adjoint.

If $M$ has non-empty boundary, then one may consider $S$ as a symmetric, densely defined operator in $L^{2}(M)$, by restricting $S$ on the space of $f \in C_{c}^{\infty}(M)$ satisfying Dirichlet $(f=0)$, Neumann $(n(f)=0)$, Robin $(n(f)+b f=0)$, or mixed boundary conditions (that is, $f=0$ on a subset of $\partial M$ and $n(f)=0$ on the rest of $\partial M)$.

(ii) Laplacian with density. Let $\mu$ be a measure on $M$ which is expressed by a positive smooth density in terms of the volume element, $d \mu=h^{2} d$ Vol. The Laplacian with respect to this density is defined by

$$
\Delta_{\mu} f=\Delta f-\frac{2}{h}\langle\operatorname{grad} h, \operatorname{grad} f\rangle,
$$

for any $f \in C^{\infty}(M)$. If $M$ has empty boundary, then the operator

$$
\Delta_{\mu}: C_{c}^{\infty}(M) \subset L^{2}(M, \mu) \rightarrow L^{2}(M, \mu)
$$

is symmetric and non-negative definite. If, in addition, $M$ is complete, then this operator is essentially self-adjoint (see, for instance [16, Theorem 2.2]).

(iii) Higher-order Laplacian. If $M$ has empty boundary, then for any $k \in \mathbb{N}$, the operator

$$
\Delta^{k}: C_{c}^{\infty}(M) \subset L^{2}(M) \rightarrow L^{2}(M)
$$

is symmetric and non-negative definite, which yields that it admits Friedrichs extension. If, in addition, $M$ is complete, then this operator is essentially selfadjoint (see for instance, [12]).

If $M$ has non-empty boundary, then we may consider the operator $\Delta^{k}$ on the space of compactly supported, smooth functions satisfying Dirichlet boundary conditions (according to the terminology of [15])

$$
\begin{aligned}
& \Delta^{k}:\left\{f \in C_{c}^{\infty}(M): f=n(f)=\cdots=n^{(k-1)}(f)=0 \text { on } \partial M\right\} \\
& \quad \subset L^{2}(M) \rightarrow L^{2}(M) .
\end{aligned}
$$

For $k=1,2$, this operator is symmetric and non-negative definite, which yields that it admits Friedrichs extension. For $k \geq 3$, this operator is not symmetric. 
However, according to Lemma 2.4, this operator is closable and Theorem 4.1 can be applied.

(iv) Laplace-type operators. Let $E \rightarrow M$ a Riemannian vector bundle endowed with a metric connection $\nabla$. The (corresponding) connection Laplacian is defined as $\Delta=\nabla^{*} \nabla$. A Laplace-type operator is an operator of the form $S=\Delta+V$, where $V \in \Gamma(\operatorname{End} E)$ and $V(x): E_{x} \rightarrow E_{x}$ is symmetric for any $x \in M$. If $M$ has empty boundary, then the operator

$$
S: \Gamma_{c}(E) \subset L^{2}(E) \rightarrow L^{2}(E)
$$

is symmetric. If, in addition, the lowest eigenvalue of $V(x)$ is bounded from below, then this operator admits Friedrichs extension. If $M$ is closed, then this operator is essentially self-adjoint (see [18]).

It is worth to point out that the Hodge Laplacian $\Delta_{k}:=d^{*} d+d d^{*}$ acting on $k$-forms, for some $0 \leq k \leq \operatorname{dim}(M)$, is a Laplace-type operator. If we consider it as

$$
\Delta_{k}: \Gamma_{c}\left(\wedge^{k} T^{*} M\right) \subset L^{2}\left(\wedge^{k} T^{*} M\right) \rightarrow L^{2}\left(\wedge^{k} T^{*} M\right),
$$

then it is symmetric and non-negative definite. If, in addition, $M$ is complete, then this operator is essentially self-adjoint (see [12]).

Another example of Laplace-type operator that is of interest in spectral theory is the Jacobi (stability) operator of a minimal submanifold. Let $\Phi: M^{m} \rightarrow N^{k}$ be a minimal isometric immersion. The Jacobi operator $J$ is a Laplace-type operator acting on sections of the normal bundle $T^{\perp} M$. Locally, if $\eta \in \Gamma\left(T^{\perp} M\right)$ and $\left\{e_{1}, \ldots, e_{m}\right\}$ is a local orthonormal frame of $M$, the Jacoby operator is given by

$$
J \eta=\Delta^{\perp} \eta-\sum_{i=1}^{m} \alpha\left(e_{i}, A_{\eta} e_{i}\right)-\sum_{i=1}^{m}\left(R\left(\eta, e_{i}\right) e_{i}\right)^{\perp}
$$

where $\Delta^{\perp}$ is the connection Laplacian corresponding to the normal connection, $\alpha$ is the second fundamental form of $\Phi, A_{\eta}$ is the Weingarten operator with respect to $\eta$, and $R$ is the curvature tensor of $N$. If $M$ is closed, then the operator

$$
J: \Gamma\left(T^{\perp} M\right) \subset L^{2}\left(T^{\perp} M\right) \rightarrow L^{2}\left(T^{\perp} M\right)
$$

is essentially self-adjoint.

In the following corollary, we denote by $\lambda_{0}\left(\Delta_{k}, M\right)$ the bottom of the spectrum of the Friedrichs extension of the Hodge Laplacian (considered as in Examples 7.1 (iv)) acting on $k$-forms on a Riemannian manifold $M$.

Corollary 7.2 Let $p: M_{2} \rightarrow M_{1}$ be an amenable Riemannian covering of manifolds without boundary. If $\lambda_{0}\left(\Delta_{k}, M_{1}\right)=0$, for some $0 \leq k \leq \operatorname{dim}\left(M_{1}\right)$, then $\lambda_{0}\left(\Delta_{k}, M_{2}\right)=0$. 
Proof Follows from Theorem 1.2 and Proposition 4.18, since the Hodge Laplacian is non-negative definite.

We now introduce the notion of renormalized Schrödinger operators, which is required in order to establish Theorem 1.5. This notion was introduced by Brooks in [7] for the Laplacian on complete manifolds without boundary.

Let $S$ be a Schrödinger operator on a possibly non-connected Riemannian manifold $M$ without boundary, and let $\varphi \in C^{\infty}(M)$ be a positive $\lambda$-eigenfunction of $S$. It is worth to point out that we do not require $\varphi$ to be square-integrable or $M$ to be complete. Let $\mu$ be the measure expressed by $\mathrm{d} \mu=\varphi^{2} \mathrm{~d}$ Vol in terms of the volume element of $M$. Consider the separable Hilbert space $L^{2}(M, \mu)$. Evidently, the map $m_{\varphi}: L^{2}(M, \mu) \rightarrow L^{2}(M)$, given by $m_{\varphi} v:=v \varphi$ is an isometric isomorphism.

The renormalized Schrödinger operator $S_{\varphi}: \mathcal{D}\left(S_{\varphi}\right) \subset L^{2}(M, \mu) \rightarrow L^{2}(M, \mu)$ is defined by $S_{\varphi} v:=m_{\varphi}^{-1}\left(S^{(F)}-\lambda\right)\left(m_{\varphi} v\right)$, for all $v \in \mathcal{D}\left(S_{\varphi}\right)$, where $S^{(F)}$ is the Friedrichs extension of $S$ and $\mathcal{D}\left(S_{\varphi}\right):=m_{\varphi}^{-1}\left(\mathcal{D}\left(S^{(F)}\right)\right)$. Clearly, the following diagram is commutative

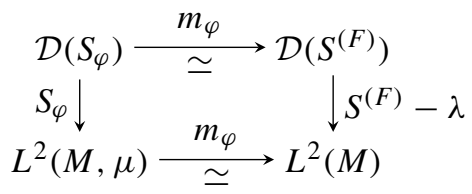

In particular, $S_{\varphi}$ is self-adjoint and $\sigma\left(S_{\varphi}\right)=\sigma(S)-\lambda$. From Proposition 2.3, it follows that

$$
\lambda_{0}\left(S_{\varphi}\right) \leq \inf _{f \in C_{c}^{\infty}(M) \backslash\{0\}} \mathcal{R}_{S_{\varphi}}(f)=\inf _{f \in C_{c}^{\infty}(M) \backslash\{0\}} \frac{\left\langle S_{\varphi} f, f\right\rangle_{L^{2}(M, \mu)}}{\|f\|_{L^{2}(M, \mu)}^{2}},
$$

Consider $\left(f_{k}\right)_{k \in \mathbb{N}} \subset C_{c}^{\infty}(M) \backslash\{0\}$, such that $\mathcal{R}_{S}\left(f_{k}\right) \rightarrow \lambda_{0}(S)$. It is evident that for $h_{k}:=m_{\varphi}^{-1}\left(f_{k}\right) \in C_{c}^{\infty}(M)$, we have $\mathcal{R}_{S_{\varphi}}\left(h_{k}\right) \rightarrow \lambda_{0}\left(S_{\varphi}\right)$. Hence, the bottom of the spectrum of $S_{\varphi}$ can be approximated with Rayleigh quotients of compactly supported smooth functions in $M$. With a simple computation of the Rayleigh quotient of such a function (as in [7, Sect. 2], using the Divergence Theorem, instead of the $*$-operator), we obtain the following expression for $\lambda_{0}(S)-\lambda$.

Proposition 7.3 Let $S$ be a Schrödinger operator on $M$ and let $\varphi \in C^{\infty}(M)$ be a positive $\lambda$-eigenfunction of $S$. Then

$$
\lambda_{0}(S)-\lambda=\inf _{f \in C_{c}^{\infty}(M) \backslash\{0\}} \frac{\int_{M}\|\operatorname{grad} f\|^{2} \varphi^{2}}{\int_{M} f^{2} \varphi^{2}} .
$$

The modified Cheeger's constant of $M$ is defined by

$$
h_{\varphi}(M):=\inf _{K} \frac{\int_{\partial K} \varphi^{2}}{\int_{K} \varphi^{2}}
$$


where the infimum is taken over all compact and smoothly bounded domains $K$ of $M$. From the preceding proposition, it is easy to establish an analog of Cheeger's inequality.

Corollary 7.4 Let $S$ be a Schrödinger operator on $M$ and let $\varphi \in C^{\infty}(M)$ be a positive $\lambda$-eigenfunction of $S$. Then

$$
\lambda_{0}(S)-\lambda \geq \frac{1}{4} h_{\varphi}(M)^{2} .
$$

Proof By virtue of Proposition 7.3, the proof is the same as that of [7, Lemma 3].

Moreover, consider the quantity

$$
h_{\varphi}^{\mathrm{ess}}(M):=\sup _{K} h_{\varphi}(M \backslash K),
$$

where the supremum is taken over all compact and smoothly bounded domains $K$ of $M$. For $\varphi=1$, this quantity is denoted by $h^{\mathrm{ess}}(M)$.

Corollary 7.5 Let $S$ be a Schrödinger operator on a complete manifold $M$ and consider a positive $\lambda$-eigenfunction $\varphi \in C^{\infty}(M)$ of $S$. Then

$$
\lambda_{0}^{\mathrm{ess}}(S)-\lambda \geq \frac{1}{4} h_{\varphi}^{\mathrm{ess}}(M)^{2} .
$$

Proof Let $\left(K_{k}\right)_{k \in \mathbb{N}}$ be an exhausting sequence of $M$, consisting of smoothly bounded domains. It is easy to see that

$$
h_{\varphi}^{\mathrm{ess}}(M)=\lim _{k} h_{\varphi}\left(M \backslash K_{k}\right)
$$

From Corollary 7.4, we have that

$$
\lambda_{0}\left(S, M \backslash K_{k}\right)-\lambda \geq \frac{1}{4} h_{\varphi}\left(M \backslash K_{k}\right),
$$

for any $k \in \mathbb{N}$. After taking the limit with respect to $k$, the statement follows from Proposition 4.23.

Remark 7.6 The above arguments can be easily modified in order to obtain analogous results for manifolds with boundary. In that case, it suffices to consider a $\lambda$-eigenfunction of $S$ which is positive and smooth only in the interior of $M$. Then, in Proposition 7.3, the infimum is taken over smooth functions with compact support in the interior of $M$.

Proof of Theorem 1.5: From Corollary 4.21, the first statement implies the second. From Corollary 4.20, the third statement follows from the second.

Assume that $\lambda_{0}\left(S_{1}\right)=\lambda_{0}^{\text {ess }}\left(S_{2}\right)$, for some Schrödinger operator $S_{1}$ on $M_{1}$. From Proposition 4.19, there exists a positive $\lambda_{0}\left(S_{1}\right)$-eigenfunction $\varphi \in C^{\infty}\left(M_{1}\right)$ of $S_{1}$, 
and its lift $\hat{\varphi} \in C^{\infty}\left(M_{2}\right)$ is a positive $\lambda_{0}\left(S_{1}\right)$-eigenfunction of $S_{2}$. From Corollary 7.5, it follows that $h_{\hat{\varphi}}^{\text {ess }}\left(M_{2}\right)=0$. Since $\varphi$ is positive and $M_{1}$ is closed, this yields that $h^{\text {ess }}\left(M_{2}\right)=0$.

Assume that $h^{\text {ess }}\left(M_{2}\right)=0$. Then $h\left(M_{2}\right)=0$ and Theorem 6.1 yields that $p$ is amenable. Assume that $p$ is finite sheeted. Then $M_{2}$ is closed. Consider a smoothly bounded domain $U$ of $M_{2}$, such that $M_{2} \backslash U$ is connected. Evidently, $M_{2} \backslash U$ is a compact manifold with boundary. It is clear that $h\left(M_{2} \backslash \bar{U}\right)=h\left(M_{2} \backslash U\right)$. From [10], it follows that $h^{\text {ess }}\left(M_{2}\right) \geq h\left(M_{2} \backslash U\right)>0$, which is a contradiction. Hence, $p$ is infinite sheeted.

Remark 7.7 In Theorem 1.5, if the covering is normal, then $\sigma\left(S_{1}\right) \neq \sigma_{\mathrm{ess}}\left(S_{2}\right)$. Indeed, if the equality holds, according to Corollary 1.3, we have $\sigma\left(S_{1}\right)=\sigma\left(S_{2}\right)$. Recall that the space of square-integrable, $\lambda_{0}\left(S_{2}\right)$-eigenfunctions of $S_{2}$ is either trivial or one-dimensional. From Corollary 1.3, it follows that $\lambda_{0}\left(S_{2}\right)$ is not an eigenvalue of the closure of $S_{2}$. From Proposition $2.1, \sigma_{\mathrm{ess}}\left(S_{2}\right)$ consists of eigenvalues of infinite multiplicity and accumulation points of $\sigma\left(S_{2}\right)$. Therefore, it follows that $\lambda_{0}\left(S_{2}\right)$ is an accumulation point of $\sigma\left(S_{2}\right)$. This is a contradiction, since $\sigma\left(S_{2}\right)=\sigma\left(S_{1}\right)$ is discrete.

For sake of completeness, we also prove the following corollary, describing the analogous properties for finite-sheeted coverings.

Corollary 7.8 Let $p: M_{2} \rightarrow M_{1}$ be a Riemannian covering with $M_{1}$ closed. Let $S_{1}$ be a Schrödinger operator on $M_{1}$ and $S_{2}$ its lift on $M_{2}$. Then the following are equivalent:

(i) $p$ is finite sheeted,

(ii) $\sigma\left(S_{1}\right) \subset \sigma\left(S_{2}\right)$ and $\sigma_{\mathrm{ess}}\left(S_{2}\right)=\emptyset$,

(iii) $\lambda_{0}\left(S_{1}\right)=\lambda_{0}\left(S_{2}\right) \notin \sigma_{\mathrm{ess}}\left(S_{2}\right)$,

(iv) $h\left(M_{2}\right)=0$ and $h^{\text {ess }}\left(M_{2}\right) \neq 0$.

Proof If the covering is finite sheeted, the inclusion of spectra follows from Corollary 4.22. In this case, $M_{2}$ is closed, which yields that the spectrum of $S_{2}$ is discrete. From Corollary 4.20, the second statement implies the third.

Assume that the third statement holds. Since $\lambda_{0}\left(S_{1}\right)=\lambda_{0}\left(S_{2}\right)$, as in the proof of Theorem 1.5, from Corollary 7.4, it follows that $h\left(M_{2}\right)=0$. From Theorem 1.5, it is clear that $h^{\mathrm{ess}}\left(M_{2}\right) \neq 0$.

Assume that the fourth statement holds. Since $h\left(M_{2}\right)=0$, from Theorem 6.1, $p$ is amenable. Since $h^{\text {ess }}\left(M_{2}\right) \neq 0$, from Theorem 1.5, it follows that $p$ is finite sheeted.

The following characterization for points of the essential spectrum of a Schrödinger operator is an immediate consequence of the Decomposition Principle.

Proposition 7.9 Let $S$ be a Schrödinger operator on a complete Riemannian manifold $M$ and let $\lambda \in \mathbb{R}$. Then $\lambda \in \sigma_{\mathrm{ess}}(S)$ if and only if there exists $\left(f_{k}\right)_{k \in \mathbb{N}} \subset C_{c}^{\infty}(M)$, with $f_{k}=0$ on $\partial M,\left\|f_{k}\right\|_{L^{2}(M)}=1,(S-\lambda) f_{k} \rightarrow 0$ in $L^{2}(M)$, and for every compact $K \subset M$, there exists $k_{0} \in \mathbb{N}$, such that supp $f_{k} \cap K=\emptyset$, for all $k \geq k_{0}$.

Our second application is motivated by [1, Corollary 3.8]. 
Theorem 7.10 Let $p: M_{2} \rightarrow M_{1}$ be a Riemannian covering with $M_{2}$ simply connected and complete. Let $S_{1}$ be a Schrödinger operator on $M_{1}$ and $S_{2}$ its lift on $M_{2}$. If there exists a compact $K \subset M_{1}$, such that the image of the fundamental group of any connected component of $M_{1} \backslash K$ in $\pi_{1}\left(M_{1}\right)$ is amenable, then $\sigma_{\mathrm{ess}}\left(S_{1}\right) \subset \sigma_{\mathrm{ess}}\left(S_{2}\right)$.

Proof Let $\lambda \in \sigma_{\text {ess }}\left(S_{1}\right)$. From Proposition 7.9, there exists $\left(f_{k}\right)_{k \in \mathbb{N}} \subset C_{c}^{\infty}(M)$, such that $f_{k}=0$ on $\partial M_{1},\left\|f_{k}\right\|_{L^{2}\left(M_{1}\right)}=1,(S-\lambda) f_{k} \rightarrow 0$ in $L^{2}\left(M_{1}\right)$, and for every compact $K_{0} \subset M_{1}$, there exists $k_{0} \in \mathbb{N}$, such that supp $f_{k} \cap K_{0}=\emptyset$, for all $k \geq k_{0}$.

Without loss of generality, we may assume that the supports of $f_{k}$ are connected, since we may restrict each $f_{k}$ to a connected component of its support and obtain a sequence with the same properties. Indeed, let $K_{i}$, with $1 \leq i \leq \ell(k)$ be the connected components of supp $f_{k}$. Since they are disjoint, it is clear that

$$
\begin{aligned}
& \frac{\left\|\left(S_{1}-\lambda\right) f_{k}\right\|_{L^{2}\left(M_{1}\right)}^{2}}{\left\|f_{k}\right\|_{L^{2}\left(M_{1}\right)}^{2}}=\frac{\sum_{i=1}^{\ell(k)}\left\|\left(S_{1}-\lambda\right)\left(\left.f\right|_{K_{i}}\right)\right\|_{L^{2}\left(M_{1}\right)}^{2}}{\sum_{i=1}^{\ell(k)}\left\|\left.f\right|_{K_{i}}\right\|_{L^{2}\left(M_{1}\right)}^{2}} \\
& \geq \min _{1 \leq i \leq \ell(k)} \frac{\left\|\left(S_{1}-\lambda\right)\left(\left.f\right|_{K_{i}}\right)\right\|_{L^{2}\left(M_{1}\right)}^{2}}{\left\|\left.f\right|_{K_{i}}\right\|_{L^{2}\left(M_{1}\right)}^{2}} .
\end{aligned}
$$

Let $i_{k}$ be index for which the right-hand side minimum is achieved. Evidently, considering the normalization (in $L^{2}\left(M_{1}\right)$ ) of the restriction of $f_{k}$ on $K_{i_{k}}$, instead of $f_{k}$, we obtain a sequence with the same properties as $\left(f_{k}\right)_{k \in \mathbb{N}}$, such that the supports are connected.

Consider a compact $K \subset M_{1}$, such that the image of the fundamental group of any connected component of $M_{1} \backslash K$ in $\pi_{1}\left(M_{1}\right)$ is amenable. Clearly, after passing to a subsequence, we may assume that the functions $f_{k}$ are supported in $M_{1} \backslash K$. Since for any $k \in \mathbb{N}$, the support of $f_{k}$ is connected, it follows that supp $f_{k} \subset U_{k}$, where $U_{k}$ is a connected component of $M_{1} \backslash K$. From the Lifting Theorem, it follows that the inclusion $U_{k} \hookrightarrow M_{1}$ can be lifted to the covering space $M_{k}^{\prime}:=M_{2} / \Gamma_{k}$, where $\Gamma_{k}$ is the image of $\pi_{1}\left(U_{k}\right)$ in $\pi_{1}\left(M_{1}\right)$. In particular, any $f_{k}$ can be lifted to some $f_{k}^{\prime} \in C_{c}^{\infty}\left(M_{k}^{\prime}\right)$.

Since the covering $q_{k}: M_{2} \rightarrow M_{k}^{\prime}$ is normal with deck transformations group $\Gamma_{k}$, it follows that it is amenable. If $q_{k}$ is finite sheeted, let $\tilde{f}_{k}$ be the normalized (in $L^{2}\left(M_{2}\right)$ ) lift of $f_{k}^{\prime}$ on $M_{2}$. If $q_{k}$ is infinite sheeted, from Proposition 4.12, there exists $\tilde{f}_{k} \in C_{c}^{\infty}\left(M_{2}\right)$, such that $\left\|\tilde{f}_{k}\right\|_{L^{2}\left(M_{2}\right)}=1$, supp $\tilde{f}_{k} \subset q_{k}^{-1}\left(\operatorname{supp} f_{k}^{\prime}\right)$, and

$$
\left\|\left(S_{2}-\lambda\right) \tilde{f}_{k}\right\|_{L^{2}\left(M_{2}\right)} \leq\left\|\left(S_{k}^{\prime}-\lambda\right) f_{k}^{\prime}\right\|_{L^{2}\left(M_{k}^{\prime}\right)}+\frac{1}{k}=\left\|\left(S_{1}-\lambda\right) f_{k}\right\|_{L^{2}\left(M_{1}\right)}+\frac{1}{k},
$$

where $S_{k}^{\prime}$ is the lift of $S_{1}$ on $M_{k}^{\prime}$. In particular, $\left(S_{2}-\lambda\right) \tilde{f}_{k} \rightarrow 0$ in $L^{2}\left(M_{2}\right)$ and supp $\tilde{f}_{k}$ is contained in $p^{-1}\left(\operatorname{supp} f_{k}\right)$. From Proposition 7.9 , it follows that $\lambda \in \sigma_{\mathrm{ess}}\left(S_{2}\right)$.

Remark 7.11 In the proof of Theorem 7.10, the only properties of Schrödinger operators used are essential self-adjointness and Proposition 7.9, which follows from the Decomposition Principle. Therefore, this proof establishes the analogous result for 
essentially self-adjoint differential operators, for which the Decomposition Principle holds (cf. [3]). For instance, if $M_{1}$ has empty boundary, then the statement of Theorem 7.10 holds for any elliptic differential operator $D_{1}$, such that $D_{1}$ and $D_{2}$ are essentially self-adjoint on the spaces of compactly supported smooth sections.

Proof of Corollary 1.6: Follows immediately from Theorem 7.10 and Corollary 4.20.

Let $p: M_{2} \rightarrow M_{1}$ be a Riemannian covering of complete manifolds, without boundary. As stated in the Introduction, there are examples where $p$ is non-amenable and $\lambda_{0}\left(M_{1}\right)=\lambda_{0}\left(M_{2}\right)$. From Theorem 1.1, Propositions 4.17 and 2.1, if $p$ is amenable, then $\sigma\left(M_{1}\right) \subset \sigma\left(M_{2}\right)$. It is natural to examine if this inclusion implies amenability of the covering. From Theorem 7.10, it is easy to construct an example of a non-amenable, normal Riemannian covering $p: M_{2} \rightarrow M_{1}$ with $M_{1}$ complete, with bounded geometry and of finite topological type (that is, $M_{1}$ admits a finite triangulation, where the simplices are defined on the standard simplex with possibly some lower-dimensional faces removed), such that $\sigma\left(M_{1}\right)=\sigma\left(M_{2}\right)$.

Example 7.12 Let $M_{1}$ be a 2-dimensional torus with a cusp, endowed with a Riemannian metric, such that $M_{1}$ is complete and outside a compact set the metric is the standard metric of the flat cylinder. It is clear that $M_{1}$ is of finite topological type and has bounded geometry. From [19, Theorem 1], it follows that $\sigma_{\text {ess }}\left(M_{1}\right)=[0,+\infty)$. Clearly, there exists a compact subset $K$ of $M_{1}$, such that $\pi_{1}\left(M_{1} \backslash K\right)=\mathbb{Z}$. From Theorem 7.10, it follows that for the simply connected covering space $M_{2}$ of $M_{1}$, we have $\sigma_{\text {ess }}\left(M_{2}\right)=[0,+\infty)$. However, $\pi_{1}\left(M_{1}\right)$ is the free group in two generators, which is non-amenable (cf. [4, Sect. 2]).

For our next application, we need the following standard lemma for the spectrum of self-adjoint operators (see, for instance, [17]).

Lemma 7.13 Let $A: \mathcal{D}(A) \subset \mathcal{H} \rightarrow \mathcal{H}$ be a self-adjoint operator on a separable Hilbert space over $\mathbb{R}$ or $\mathbb{C}$. Assume that for some $\lambda \in \mathbb{R}$ and $\varepsilon>0$, there exists $v \in \mathcal{D}(A)$, with $\|v\|=1$ and $\|(A-\lambda) v\|<\varepsilon$. Then there exists $\lambda^{\prime} \in \sigma(A)$, such that $\left|\lambda-\lambda^{\prime}\right|<\varepsilon$.

Proposition 7.14 Let $M$ be a closed manifold with infinite, amenable, and residually finite fundamental group. Then the spectrum of the Laplacian on the universal covering space $\tilde{M}$ of $M$ is given by

$$
\sigma(\tilde{M})=\sigma_{\mathrm{ess}}(\tilde{M})=\overline{\cup_{\alpha} \sigma\left(M_{\alpha}\right)}
$$

where the union is taken over all finite-sheeted covering spaces $M_{\alpha}$ of $M$.

Proof Since $\pi_{1}(M)$ is infinite, from Corollary 1.3, it follows that $\sigma(\tilde{M})=\sigma_{\text {ess }}(\tilde{M})$. Since $p: \tilde{M} \rightarrow M$ is infinite sheeted and amenable, from Theorem 1.5, it follows that $\lambda_{0}^{\text {ess }}(\tilde{M})=0$. Let $M_{\alpha}$ be a finite-sheeted covering space of $M$. Since $M_{\alpha}$ is closed and 
$\lambda_{0}^{\text {ess }}(\tilde{M})=0$, from Theorem 1.5 (applied to the covering $p_{\alpha}: \tilde{M} \rightarrow M_{\alpha}$ ), it follows that $\sigma\left(M_{\alpha}\right) \subset \sigma_{\text {ess }}(\tilde{M})$. Since $\sigma(\tilde{M})$ is closed, this yields that

$$
\overline{\cup_{\alpha} \sigma\left(M_{\alpha}\right)} \subset \sigma(M)
$$

where the union is taken over all finite-sheeted covering spaces $M_{\alpha}$ of $M$.

Consider $\lambda \in \sigma(\tilde{M})$ and $\varepsilon>0$. From Proposition 2.1 and Lemma 2.2, there exists $f \in C_{c}^{\infty}(\tilde{M})$, with $\|f\|_{L^{2}(\tilde{M})}=1$ and $\|(\Delta-\lambda) f\|_{L^{2}(\tilde{M})}<\varepsilon$. Since $\pi_{1}\left(M_{1}\right)$ is residually finite, for any compact $K \subset \tilde{M}$, there exists a finite-sheeted covering space $M_{\alpha}$ of $M$, such that the covering $p_{\alpha}: \tilde{M} \rightarrow M_{\alpha}$ restricted on $K$ is injective (see for instance [8]). In particular, there exists a finite-sheeted covering space $M_{\alpha}$ of $M$, such that the covering $p_{\alpha}: \tilde{M} \rightarrow M_{\alpha}$ restricted in a compact neighborhood $K$ of supp $f$ is an isometry onto its image. Consider the function $f_{\alpha}:=\left.f \circ p_{\alpha}\right|_{K} ^{-1}$ extended by zero outside $p_{\alpha}(K)$. Evidently, $f_{\alpha} \in C^{\infty}\left(M_{\alpha}\right)$ and satisfies

$$
\left\|f_{\alpha}\right\|_{L^{2}\left(M_{\alpha}\right)}=1 \text { and }\left\|(\Delta-\lambda) f_{\alpha}\right\|_{L^{2}\left(M_{\alpha}\right)}<\varepsilon .
$$

From Lemma 7.13, it follows that there exists $\lambda^{\prime} \in \sigma\left(M_{\alpha}\right)$ with $\left|\lambda-\lambda^{\prime}\right|<\varepsilon$. Since $\varepsilon>0$ is arbitrary, this establishes the asserted equality.

Recall that the spectrum of the Laplacian on a closed Riemannian manifold $M$ is discrete; that is, it consists of isolated eigenvalues

$$
0=\lambda_{0}(M)<\lambda_{1}(M)<\lambda_{2}(M)<\ldots
$$

of finite multiplicity. From the above proposition, we can easily recover the following observation of Sunada [24], which was also established by Brooks [8, Theorem 2].

Corollary 7.15 Let $M$ be a closed manifold with infinite, amenable, and residually finite fundamental group. Then there exists a sequence $\left(M_{i}\right)_{i \in \mathbb{N}}$ of finite-sheeted covering spaces of $M$, such that $\lambda_{1}\left(M_{i}\right) \rightarrow 0$, as $i \rightarrow+\infty$.

Proof From Proposition 7.14, it follows that $\lambda_{0}^{\text {ess }}(\tilde{M})=0$, where $\tilde{M}$ is the universal covering space of $M$. Since $p: \tilde{M} \rightarrow M$ has infinite deck transformations group, from Corollary 5.4, we obtain that zero is an accumulation point of $\sigma(\tilde{M})$. From Proposition 7.14, it follows that there exist finite-sheeted covering spaces $M_{i}$ of $M$, with $i \in \mathbb{N}$, and $k_{i} \in \mathbb{N}$, such that $\lambda_{k_{i}}\left(M_{i}\right) \rightarrow 0$, as $i \rightarrow+\infty$. Since $0<\lambda_{1}\left(M_{i}\right) \leq \lambda_{k_{i}}\left(M_{i}\right)$, for any $i \in \mathbb{N}$, this completes the proof.

We now present some examples of amenable coverings. The following observation, provides a sufficient geometric condition for amenability of coverings.

Proposition 7.16 Let $M_{1}$ be a complete Riemannian manifold, without boundary and with non-negative Ricci curvature. Then any covering $p: M_{2} \rightarrow M_{1}$ is amenable.

Proof Let $\tilde{M}$ be the simply connected covering space of $M_{1}$. From the Bishop-Gromov Comparison Theorem, it follows that $\tilde{M}$ has polynomial growth and hence, every 
finitely generated subgroup of $\pi_{1}\left(M_{1}\right)$ has polynomial growth (cf. [20]). From Corollary 2.9, it follows that every finitely generated subgroup of $\pi_{1}\left(M_{1}\right)$ is amenable and Corollary 2.10 yields that so is $\pi_{1}\left(M_{1}\right)$. Therefore, any covering $p: M_{2} \rightarrow M_{1}$ is amenable.

Example 7.17 Let $M$ be a Riemannian manifold and denote by $\tilde{M}$ its universal covering space. The homology cover of $M$ is defined by

$$
M_{H}:=\tilde{M} /\left[\pi_{1}(M), \pi_{1}(M)\right] .
$$

Evidently, the Riemannian covering $p: M_{H} \rightarrow M$ is normal with deck transformations group

$$
\Gamma=\pi_{1}(M) /\left[\pi_{1}(M), \pi_{1}(M)\right]=H_{1}(M) .
$$

Since $H_{1}(M)$ is abelian, from Corollary 2.11, it follows that $p: M_{H} \rightarrow M$ is amenable.

Next, we present an example of an infinite-sheeted amenable covering with trivial deck transformations group. In particular, this implies that the results of Sect. 5 cannot be applied to arbitrary infinite-sheeted amenable coverings.

Example 7.18 Let $\Gamma_{1}$ be the countable group of invertible, upper triangular $2 \times 2$ matrices with entries in $\mathbb{Q}$ and let $M_{1}$ be a Riemannian manifold with $\pi_{1}\left(M_{1}\right)=\Gamma_{1}$ (cf. [2, Sect. 5]). Let $\Gamma_{2}$ be the subgroup of $\Gamma_{1}$ consisting of diagonal matrices. Denote by $\tilde{M}$ the simply connected covering space of $M_{1}$ and consider $M_{2}:=\tilde{M} / \Gamma_{2}$. It is easy to see that the covering $p: M_{2} \rightarrow M_{1}$ is infinite sheeted and does not have non-trivial deck transformations. However, $\Gamma_{1}$ is solvable and in particular, amenable (from Corollary 2.11), which yields that $p$ is an amenable covering.

Recall that in our main results there are no assumptions on the vector bundles, the connections, and the differential operators. The next example demonstrates that these play a crucial role in the behavior of the spectrum even under finite-sheeted coverings. Namely, this example shows that whether or not the bottom of the spectrum of the connection Laplacian is preserved under a Riemannian covering depends on the corresponding metric connection. Moreover, this example demonstrates that the inequality of Corollary 4.20, which holds for Schrödinger operators, is not true (in general) for the connection Laplacian.

Let $M$ be a complete Riemannian manifold and $E \rightarrow M$ a Riemannian vector bundle endowed with a metric connection $\nabla$. The corresponding connection Laplacian $\Delta$ (considered as in Examples 7.1(iv)) is essentially self-adjoint (cf. [18]). In the following example, we denote by $\lambda_{0}(\Delta, E)$ the bottom of the spectrum of its closure. It is worth to point out that if $M$ is closed, then the spectrum of this operator is discrete (cf. [18]).

Example 7.19 Consider $S_{1}:=\mathbb{R} / \mathbb{Z}$ and the trivial bundle $E_{1}:=S_{1} \times \mathbb{R}^{2}$ with the standard metric. We can identify smooth sections of $E_{1}$ with smooth maps $f: \mathbb{R} \rightarrow \mathbb{R}^{2}$ 
with $f(x)=f(x+1)$, for all $x \in \mathbb{R}$. For $\phi \in \mathbb{R}$, consider the metric connection $\nabla^{\phi}$, defined by

$$
\nabla_{\frac{d}{d x}}^{\phi} f(x):=\left(\begin{array}{cc}
\cos (x \phi) & -\sin (x \phi) \\
\sin (x \phi) & \cos (x \phi)
\end{array}\right) \frac{d}{d x}\left(\begin{array}{cc}
\cos (x \phi) & \sin (x \phi) \\
-\sin (x \phi) & \cos (x \phi)
\end{array}\right)\left(\begin{array}{l}
f_{1}(x) \\
f_{2}(x)
\end{array}\right)
$$

for any smooth section $f=\left(f_{1}, f_{2}\right)$ of $E_{1}$. Since the spectrum of the connection Laplacian $\Delta^{\phi}$ is discrete for any $\phi \in \mathbb{R}$, it is clear that $\lambda_{0}\left(\Delta^{\phi}, E_{1}\right)=0$ if and only if there exists a parallel section of $E_{1}$ with respect to $\nabla^{\phi}$, or equivalently, $\phi=2 k \pi$, for some $k \in \mathbb{Z}$.

For $k \in \mathbb{N} \backslash\{1\}$, consider a $k$-sheeted Riemannian covering $p_{k}: S_{1}^{(k)} \rightarrow S_{1}$ and the pullback bundle $E_{2}$ of $E_{1}$ endowed with the standard metric and the pullback connection $\nabla^{\phi}$. It is clear that $\lambda_{0}\left(\Delta^{2 \pi}, E_{2}\right)=\lambda_{0}\left(\Delta^{2 \pi}, E_{1}\right)=0$. However, the above arguments imply that $\lambda_{0}\left(\Delta^{2 \pi / k}, E_{2}\right)=0<\lambda_{0}\left(\Delta^{2 \pi / k}, E_{1}\right)$.

This is an example of a finite-sheeted covering which shows that the inequality of Corollary 4.20 does not hold for the connection Laplacian. Based on this example, it is easy to construct an analogous example of an infinite-sheeted covering. Consider the covering $p: \mathbb{R} \rightarrow S_{1}^{(k)}$ and the pullback bundle $E$ of $E_{2}$ endowed with the standard metric and the pullback connection $\nabla^{2 \pi / k}$. Since $p$ is infinite sheeted and amenable, from Theorem 1.2, since the connection Laplacian is non-negative definite, it follows that $\lambda_{0}\left(\Delta^{2 \pi / k}, E\right)=\lambda_{0}^{\text {ess }}\left(\Delta^{2 \pi / k}, E\right)=0<\lambda_{0}\left(\Delta^{2 \pi / k}, E_{1}\right)$.

A natural question arising from our results is whether it is possible to obtain an analog of Theorem 1.1 for Friedrichs extensions of operators (that is, in the context of Theorem 1.2). It is worth to point out that this holds the Laplacian on manifolds which are isometric to the interior of complete manifolds with boundary. Indeed, in such case, the spectrum of the Friedrichs extension of the Laplacian in the interior coincides with the Dirichlet spectrum of the Laplacian on the manifold with boundary. Since the latter one is essentially self-adjoint, the inclusion of the spectra follows from Theorem 1.1.

Moreover, during the last years, there is a lot of progress in the study of the Dirichletto-Neumann spectrum. Although the Dirichlet-to-Neumann map is not a differential operator, there are interesting relations between its spectrum and the geometry and topology of the underlying manifold. Therefore, it is natural to ask whether similar results hold for its behavior under Riemannian coverings. This is indeed the case. However, the methods to establish them are quite different. Therefore, we will deal with this in a forthcoming paper.

Acknowledgements Open access funding provided by Max Planck Society. I would like to thank Werner Ballmann and Dorothee Schüth for some very enlightening discussions and helpful remarks. I am also grateful to the Max Planck Institute for Mathematics in Bonn for its support and hospitality. I would also like to thank the referees for their helpful comments.

Open Access This article is distributed under the terms of the Creative Commons Attribution 4.0 International License (http://creativecommons.org/licenses/by/4.0/), which permits unrestricted use, distribution, and reproduction in any medium, provided you give appropriate credit to the original author(s) and the source, provide a link to the Creative Commons license, and indicate if changes were made. 


\section{References}

1. Ballmann, W., Matthiesen, H., Mondal, S.: Small eigenvalues of surfaces of finite type. arXiv: 1506.06541

2. Ballmann, W., Matthiesen, H., Polymerakis, P.: On the bottom of spectra under coverings. Math. Z. 288(3-4), 1029-1036 (2018)

3. Bär, C.: The Dirac operator on hyperbolic manifolds of finite volume. J. Differ. Geom. 54(3), 439-488 (2000)

4. Bérard, P., Castillon, P.: Spectral positivity and Riemannian coverings. Bull. Lond. Math. Soc. 45(5), 1041-1048 (2013)

5. Bessa, G.P., Montenegro, J.F., Piccione, P.: Riemannian submersions with discrete spectrum. J. Geom. Anal. 22(2), 603-620 (2012)

6. Brooks, R.: The fundamental group and the spectrum of the Laplacian. Comment. Math. Helv. 56(4), 581-598 (1981)

7. Brooks, R.: The bottom of the spectrum of a Riemannian covering. J. Reine Angew. Math. 357, 101-114 (1985)

8. Brooks, R.: The spectral geometry of a tower of coverings. J. Differ. Geom. 23(1), 97-107 (1986)

9. Buser, P.: A note on the isoperimetric constant. Ann. Sci. École Norm. Sup. (4) 15(2), 213-230 (1982)

10. Cheeger, J.: A Lower Bound for the Smallest Eigenvalue of the Laplacian, Problems in Analysis (Papers Dedicated to Salomon Bochner, 1969). Princeton University Press, Princeton, pp. 195-199 (1970)

11. Cheng, S.Y., Yau, S.T.: Differential equations on Riemannian manifolds and their geometric applications. Commun. Pure Appl. Math. 28(3), 333-354 (1975)

12. Chernoff, P.R.: Essential self-adjointness of powers of generators of hyperbolic equations. J. Funct. Anal. 12, 401-414 (1973)

13. Donnelly, H.: On the essential spectrum of a complete Riemannian manifold. Topology 20(1), 1-14 (1981)

14. Fischer-Colbrie, D., Schoen, R.: The structure of complete stable minimal surfaces in 3-manifolds of nonnegative scalar curvature. Commun. Pure Appl. Math. 33(2), 199-211 (1980)

15. Gazzola, F., Grunau, H.-C., Sweers, G.: Polyharmonic boundary value problems. In: Positivity Preserving and Nonlinear Higher Order Elliptic Equations in Bounded Domains. Lecture Notes in Mathematics, p. 2010. Springer, Berlin (1991)

16. Grigor'yan, A.: Heat kernels on weighted manifolds and applications, the ubiquitous heat kernel. Contemp. Math. 398, 93-191 (2006)

17. Hislop, P.D., Sigal, I.M.: Introduction to spectral theory. With applications to Schrödinger operators. In: Applied Mathematical Sciences, vol. 113. Springer, New York (1996)

18. Lawson Jr., H.B., Michelsohn, M.-I.: Spin Geometry. Princeton Mathematical Series, vol. 38. Princeton University Press, Princeton (1989)

19. Lu, Z., Zhou, D.: On the essential spectrum of complete non-compact manifolds. J. Funct. Anal. 260(11), 3283-3298 (2011)

20. Milnor, J.: A note on curvature and fundamental group. J. Differ. Geom. 2, 1-7 (1968)

21. Nomizu, K., Ozeki, H.: The existence of complete Riemannian metrics. Proc. Am. Math. Soc. 12, 889-891 (1961)

22. Roblin, T., Tapie, S.: Exposants critiques et moyennabilité, (French), Géométrie ergodique, Monogr. Enseign. Math., 43, Enseignement Math., Geneva, pp. 61-92 (2013)

23. Sullivan, D.: Related aspects of positivity in Riemannian geometry. J. Differ. Geom. 25(3), 327-351 (1987)

24. Sunada, T.: Riemannian coverings and isospectral manifolds. Ann. Math. (2) 121(1), 169-186 (1985)

25. Taylor, M.E.: Partial Differential Equations I. Basic Theory (Applied Mathematical Sciences), vol. 115, 2nd edn. Springer, New York (2011)

Publisher's Note Springer Nature remains neutral with regard to jurisdictional claims in published maps and institutional affiliations. 